AperTO - Archivio Istituzionale Open Access dell'Università di Torino

\title{
Radial solutions of Dirichlet problems with concave-convex nonlinearities
}

\section{This is the author's manuscript}

Original Citation:

Availability:

This version is available http://hdl.handle.net/2318/86696

since 2015-09-21T08:15:01Z

Terms of use:

Open Access

Anyone can freely access the full text of works made available as "Open Access". Works made available under a Creative Commons license can be used according to the terms and conditions of said license. Use of all other works requires consent of the right holder (author or publisher) if not exempted from copyright protection by the applicable law. 


\section{UNIVERSITÀ DEGLI STUDI DI TORINO}

This Accepted Author Manuscript (AAM) is copyrighted and published by Elsevier. It is posted here by agreement between Elsevier and the University of Turin. Changes resulting from the publishing process - such as editing, corrections, structural formatting, and other quality control mechanisms may not be reflected in this version of the text. The definitive version of the text was subsequently published in [Nonlinear Analysis: theory, methods and applications, 74, 7, 2011,

10.1016/j.na.2010.12.026].

You may download, copy and otherwise use the AAM for non-commercial purposes provided that your license is limited by the following restrictions:

(1) You may use this AAM for non-commercial purposes only under the terms of the CC-BY-NC-ND license.

(2) The integrity of the work and identification of the author, copyright owner, and publisher must be preserved in any copy.

(3) You must attribute this AAM in the following format: Creative Commons BY-NC-ND license (http://creativecommons.org/licenses/by-nc-nd/4.0/deed.en), [10.1016/j.na.2010.12.026 -

http://www.journals.elsevier.com/nonlinear-analysis-theory-methods-and-applications/] 


\title{
Radial solutions of Dirichlet problems with concave-convex nonlinearities *
}

\author{
Francesca Dalbono \\ CMAF, Universidade de Lisboa, \\ Av. Prof. Gama Pinto 2, 1649-003 Lisboa, Portugal \\ email: fdalbono@ptmat.fc.ul.pt \\ Walter Dambrosio \\ Dipartimento di Matematica, Università di Torino, \\ Via Carlo Alberto 10, 10123 Torino, Italia \\ email:
}

\begin{abstract}
We prove the existence of a double infinite sequence of radial solutions for a Dirichlet concave-convex problem associated with an elliptic equation in a ball of $\mathbb{R}^{n}$. We are interested in relaxing the classical positivity condition on the weights, by allowing the weights to vanish. The idea is to develop a topological method and to use the concept of rotation number. The solutions are characterized by their nodal properties.
\end{abstract}

\section{Introduction}

In this paper we are concerned with the Dirichlet problem

$$
\begin{cases}\Delta u(x)+q(|x|)|u(x)|^{\delta-1} u(x)+p(|x|)|u(x)|^{\gamma-1} u(x)=0 & x \in \Omega \\ u(x)=0 & x \in \partial \Omega\end{cases}
$$

where $\Omega$ is the unit ball in $\mathbb{R}^{N}$ with $N \geq 3$ and $p, q:[0,1] \rightarrow \mathbb{R}$ are $C^{1}$ functions. Moreover, we assume $0<\gamma<1<\delta$; hence, the nonlinearity we are dealing with is of concave-convex type.

We are interested in the search of nodal solutions of (1.1) with prescribed nodal properties. This kind of problem has been intensively studied in literature; for a quite exhaustive bibliography, we refer to [6], [9], [19], [33] and references therein.

The nonlinearity we are studying combines two different aspects; on the one hand it is superlinear at infinity, on the other hand it is sublinear near zero. As a consequence,

${ }^{*} 2000$ Mathematics Subject Classification: 34B15. 
the problem inherits both the qualitative features of superlinear and sublinear problems. Starting from the pioneering paper [3], many authors have studied this problem, under various assumptions on the coefficients $q$ and $p$. The main question is to prove the existence of four sequences of nodal solutions of (1.1), two of them with large norm and two with small norm.

This has been proved, for instance, in [9] and [21, Theorem 1.5] when $q$ and $p$ are strictly positive. In the one-dimensional setting, we wish to quote, among others, the contribution of [1], [11] and [29, Theorem 4], providing a detailed study of the exact structure of the solutions in presence of positive constant weights.

The paper [1] treats also the $p$-strictly-negative case, showing that, in this context, no more than two sequences of solutions can be achieved. More in general, the positiveness of each weight on a subset of $\Omega$ of non-zero measure is a necessary condition to guarantee the existence of four sequences of solutions. It is still an open question whether this condition is also sufficient.

As for multiplicity of positive solutions, in the more general case of nonradial weights $q$ and $p$, an answer to this question has been recently obtained in [7], [15, Corollary 2.2], [16, Theorem 4.2] and [17, Theorem 6]. These papers provide the existence of two positive solutions in the case where the weights are allowed to vanish and change sign in $\Omega$. We wish to remark that there is an extensive literature concerning multiplicity results of positive solutions to problem (1.1) under relaxed sign assumptions on one of the weights (cf., for instance [2], [18, Theorem 6.9], [21, Theorem 1.1], [23], [27], [37], [41]). For interesting extensions to concave-convex systems, we refer to [34, Theorem $1.2(\mathrm{~d})]$.

Focusing our attention on the superlinear and sub-critical structure of problem (1.1) and taking into account the wide literature about superlinear nonlinearities at infinity (cf., among others, the classical paper [38]), we immediately deduce the existence of two sequences of large norm solutions for (1.1), provided that $q$ is positive, regardless of the sign of the weight $p$. In this setting, we quote, among many others, the papers $[21$, Theorem 1.5 (I)-(ii)], [26], [28], [31], [36].

In the superlinear context, interesting multiplicity results have been also achieved under relaxed sign conditions on the weight $q$. In particular, we refer to [13], dealing with a possibly vanishing coefficient $q$ and to [5], [39] and reference therein, dealing with nonradial and indefinite (changing sign) weights. More in detalis, in [5] $q$ is supposed to have a "thick" zero set, while in [39] the authors assume that $\nabla q(x) \neq 0$ for every $x \in \Omega$ with $q(x)=0$.

Analogously, privileging the sublinear aspect of (1.1), many authors have proved the existence of two sequences of small norm solutions, provided that $p$ is positive, regardless of the sign of $q$ (cf., among many others, [9], [22], [25], [40], [42, Theorem 3.3]).

The list of result available in literature concered with relaxed sign condition on the coefficient $p$ in a sublinear context is shorter. Some existence result in presence of indefinite weights can be found in [4] and [32, Theorem 2.3]. We wish also to mention the work [30] concerned with multiple solutions to the equation (1.1) in $\mathbb{R}^{n}$ when $p$ is 
allowed to vanish.

Our aim consists in proving the existence of changing sign solutions in the case where the coefficients $q$ and $p$ can vanish.

In particular, we assume that

$$
q \succ 0, \quad p \succ 0,
$$

where, given a continuous function $\varphi:[0,1] \rightarrow \mathbb{R}$, by $\varphi \succ 0$ we mean that $\varphi(r) \geq 0$ for every $r \in[0,1]$ and $\varphi \not \equiv 0$.

According to [13], we also require that both $q$ and $p$ satisfy some regularity conditions in a neighbourhood of $r=0$ and of their zeros. More precisely, we first suppose that one of the following alternatives holds

$$
\begin{gathered}
q(0)>0 \\
\text { or } \\
\exists \beta>0, h \geq 1: q(r) \sim \beta r^{h}, \quad \text { as } r \rightarrow 0^{+} .
\end{gathered}
$$

Analogously, we assume that

$$
\begin{gathered}
p(0)>0 \\
\text { or } \\
\exists \alpha>0, k \geq 1: p(r) \sim \alpha r^{k}, \quad \text { as } r \rightarrow 0^{+} .
\end{gathered}
$$

Finally, denoting by $\mathcal{C}_{0}$ and $\mathcal{C}_{1}$ the set of zeros of $q$ and $p$ in $(0,1]$, respectively, i.e.

$$
\mathcal{C}_{0}:=\{r \in(0,1]: q(r)=0\} \quad \text { and } \quad \mathcal{C}_{1}:=\{r \in(0,1]: p(r)=0\},
$$

we assume that

$\mathcal{C}_{0}$ and $\mathcal{C}_{1}$ have a finite number of connected components,

denoted respectively by $I_{i}=\left[\widetilde{r}_{i}, r_{i}\right]$ and $J_{j}=\left[\widetilde{s}_{j}, s_{j}\right]$ with $i \in\{1, \ldots, n\}, j \in\{1, \ldots, m\}$;

$$
\begin{array}{ll}
q^{\prime} \leq 0 & \text { in a left neighborhood of } \widetilde{r}_{i}, \\
q^{\prime} \geq 0 & \text { in a right neighborhood of } r_{i}, \\
p^{\prime} \leq 0 & \text { in a left neighborhood of } \widetilde{s}_{i}, \\
p^{\prime} \geq 0 & \text { in a right neighborhood of } s_{i} .
\end{array}
$$

Our purpose will be attained in two steps; first of all, we will concentrate in the search of solutions with large $C^{1}$-norm. In this context, one more assumption involving the superlinear term is needed. More precisely, we require the nonlinearity to be subcritical by setting

$$
\delta<\frac{N+2}{N-2}, \quad \text { if condition }(1.3) \text { is satisfied. }
$$


Condition (1.12) can be relaxed by assuming

$$
\delta<\frac{N+2+2 h}{N-2}, \quad \text { if condition (1.4) holds. }
$$

Following [13], by means of a shooting approach, we are able to prove the following result

Theorem 1.1 Consider $p, q \in C^{1}([0,1], \mathbb{R})$ satisfying (1.2), and one of the alternatives (1.3)-(1.6) associated with (1.12)-(1.13). Moreover, assume (1.7)-(1.11). Then, there exists $j_{0} \in \mathbb{N}$ such that for every integer $n \geq j_{0}$ there exist two radial solutions $u_{n}$ and $v_{n}$ of $(1.1)$, satisfying $u_{n}(0)>1$ and $v_{n}(0)<-1$ and

$$
\lim _{n \rightarrow+\infty}\left\|u_{n}\right\|_{1}=+\infty, \quad \lim _{n \rightarrow+\infty}\left\|v_{n}\right\|_{1}=+\infty .
$$

On the other hand, in the spirit of [9], we can prove the validity of the following

Theorem 1.2 Consider $p, q \in C^{1}([0,1], \mathbb{R})$ satisfying (1.2), and one of the alternatives (1.3)-(1.6) associated with (1.12)-(1.13). Moreover, assume (1.7)-(1.11). Then, there exists $j_{0} \in \mathbb{N}$ such that for every integer $n \geq j_{0}$ there exist two radial solutions $z_{n}$ and $w_{n}$ of $(1.1)$, satisfying $0<z_{n}(0)<1$ and $-1<w_{n}(0)<0$.

As a consequence, we get the existence of four sequences of solutions of (1.1).

We remark that the same result can be obtained for the more general problem

$$
\begin{cases}\Delta u(x)+q(|x|) g(u)+p(|x|) f(u)=0 & x \in \Omega \\ u(x)=0 & x \in \partial \Omega .\end{cases}
$$

Indeed, we can prove results analogous to Theorem 1.1 and Theorem 1.2 for (1.14) when $f \in C(\mathbb{R})$ satisfies

$$
\lim _{u \rightarrow 0} \frac{f(u)}{u}=+\infty
$$

and $g \in C(\mathbb{R})$ fulfills

$$
\lim _{|u| \rightarrow+\infty} \frac{g(u)}{u}=+\infty
$$

and a suitable sub-critical growth condition at infinity (see Theorem 2.1 and Theorem 2.4 in [13] for the details on the precise assumptions on $g$ ).

In the proof, for the sake of simplicity, we will consider the easier problem (1.1).

\section{Preliminary notation}

We focus our attention on the boundary value problem

$$
\left\{\begin{array}{l}
\left(r^{N-1} u^{\prime}(r)\right)^{\prime}+r^{N-1}|u(r)|^{\delta-1} u(r) q(r)+r^{N-1}|u(r)|^{\gamma-1} u(r) p(r)=0 \\
u^{\prime}(0)=0=u(1)
\end{array}\right.
$$


where $p, q \in C^{1}([0,1], \mathbb{R}), N \geq 3$ and $0<\gamma<1<\delta$. We are interested in the search of its solutions, since, by setting $r=|x|$, it is immediate to notice that each solution to (2.1) is a radial solution of the Dirichlet problem (1.1).

To this purpose, let us concentrate on the following Cauchy problem

$$
\left\{\begin{array}{l}
\left(r^{N-1} u^{\prime}(r)\right)^{\prime}+r^{N-1}|u(r)|^{\delta-1} u(r) q(r)+r^{N-1}|u(r)|^{\gamma-1} u(r) p(r)=0 \\
u^{\prime}(0)=0, \quad u(0)=d,
\end{array}\right.
$$

where $d$ varies in $\mathbb{R}$. We denote by $u_{d}$ a solution of $(2.2)$ and by $\left[0, \rho_{u_{d}}\right) \subset[0,1]$ its maximal interval of definition.

We devote the following sections to prove the global existence of solutions for problem (2.2), some uniqueness result and the validity of some "elastic properties".

Our idea consists in generalizing the techniques developed in [13] to the case where the presence of a sublinear term is allowed. To this aim, let us first introduce the constant $R_{1}<1$ (depending on our assumptions (1.3)-(1.6)) by the following remark

Remark 2.1 From condition (1.3) combined with the regularity assumptions on $q$, we infer the existence of $\varepsilon>0, q_{0}>0, R_{1} \in(0,1)$, such that

$$
\begin{array}{cc}
\frac{r q^{\prime}(r)}{q(r)} \geq-\varepsilon & \forall r \in\left(0, R_{1}\right], \\
q(r) \geq q_{0} & \forall r \in\left[0, R_{1}\right] .
\end{array}
$$

Furthermore, from condition (1.4), we infer the existence of $\varepsilon \in(0,1), R_{1} \in(0,1)$ such that

$$
h-\varepsilon \leq \frac{r q^{\prime}(r)}{q(r)} \leq h+\varepsilon, \quad \forall r \in\left(0, R_{1}\right]
$$

Analogously, according to assumption (1.5), it is not restrictive to choose $\varepsilon>0, p_{0}>0$, $R_{1} \in(0,1)$, such that

$$
\begin{array}{cc}
\frac{r p^{\prime}(r)}{p(r)} \geq-\varepsilon & \forall r \in\left(0, R_{1}\right], \\
p(r) \geq p_{0} & \forall r \in\left[0, R_{1}\right] .
\end{array}
$$

Furthermore, according to condition (1.6), it is not restrictive to choose $\varepsilon \in(0,1)$, $R_{1} \in(0,1)$ such that

$$
k-\varepsilon \leq \frac{r p^{\prime}(r)}{p(r)} \leq k+\varepsilon, \quad \forall r \in\left(0, R_{1}\right]
$$


Condition (2.5) and (2.8) guarantee respectively that $q$ and $p$ are strictly increasing in $\left(0, R_{1}\right]$. Hence, recalling (2.4) and (2.7), we conclude that the alternatives in (1.3)-(1.6) ensure the existence of $q_{0}>0$ and $p_{0}>0$ such that

$$
q(r) \geq q_{0} \quad \text { and } \quad p(r) \geq p_{0} \quad \forall r \in\left[R_{0}, R_{1}\right] .
$$

where $R_{0} \in\left(0, R_{1}\right)$ is fixed.

We remark that the definitions of $\varepsilon, q_{0}, R_{1}$ should be further refined. The additional refinements are described in the next part of this section and in the proofs of the main lemmas of Section 5 (cf. (5.5)-(5.6), (5.9)-(5.10) when $h>k$, and (5.12)-(5.13)).

Note that conditions (1.12) and (1.13) are respectively equivalent to

$$
\frac{N-2}{2}<\frac{N}{\delta+1} \text { and } \quad \frac{N-2}{2}<\frac{N+h}{\delta+1} .
$$

We point out that $\gamma<1<\frac{N+2}{N-2}$ or, equivalently, $\frac{N-2}{2}<\frac{N}{\gamma+1}$.

Without loss of generality, according to the previous inequalities, we can choose $\varepsilon>0$ and $R_{1} \in(0,1)$ in Remark 2.1 satisfying also

$$
\begin{gathered}
\frac{N-2}{2}<\frac{N-\varepsilon}{\delta+1} \quad \text { under assumption (1.12) } \\
\frac{N-2}{2}<\frac{N+h-\varepsilon}{\delta+1} \text { and } \frac{N-2}{2}<\frac{N-\varepsilon}{\gamma+1} \quad \text { under assumption (1.13). }
\end{gathered}
$$

Before proceeding, we need to introduce some useful notation. We define the following positive constants

$$
\begin{aligned}
Q & :=\max _{r \in[0,1]} q(r), \quad P:=\max _{r \in[0,1]} p(r) \\
Q^{\prime} & :=\max _{r \in[0,1]}\left|q^{\prime}(r)\right|, \quad P^{\prime}:=\max _{r \in[0,1]}\left|p^{\prime}(r)\right| .
\end{aligned}
$$

Given $\varphi \in C([0,1], \mathbb{R})$ with $\varphi \succ 0$, let us set

$$
\Phi_{\varphi}(r):=\int_{0}^{r} s^{N-1} \varphi(s) d s, \quad \Psi_{\varphi}(r):=\int_{0}^{r} \frac{\int_{0}^{s} t^{N-1} \varphi(t) d t}{s^{N-1}} d s \quad r \in(0,1] .
$$

It is immediate to note that $\Phi_{\varphi}$ is nondecreasing in $[0,1]$. Moreover, if $\varphi$ is positive on a right neighbourhood of 0 , then $\Psi_{\varphi}(r)$ is striclty increasing and, consequently, invertible in $(0,1]$. Observe also that, as $r \rightarrow 0^{+}$,

$$
\begin{gathered}
\Phi_{q}(r) \sim \frac{q(0)}{N} r^{N}, \quad \Psi_{q}(r) \sim \frac{q(0)}{2 N} r^{2}, \quad \text { under assumption (1.3), } \\
\Phi_{q}(r) \sim \frac{\beta r^{h+N}}{h+N}, \quad \Psi_{q}(r) \sim \frac{\beta r^{h+2}}{(h+N)(h+2)}, \quad \text { under assumption (1.4), } \\
\Phi_{p}(r) \sim \frac{\alpha r^{k+N}}{k+N}, \quad \Psi_{p}(r) \sim \frac{\alpha r^{k+2}}{(k+N)(k+2)}, \quad \text { under assumption (1.6). }
\end{gathered}
$$




\section{Some properties of the solutions in the ball $|x| \leq R_{1}$}

Our first purpose consists in describing some properties of the solutions to the initial value problem $(2.2)$ in the interval $\left[0, R_{1}\right]$. The approach followed is based on the one adopted in [13], [10] and [19].

Lemma 3.1 Consider $R_{0} \in\left(0, R_{1}\right)$ and $p, q \in C^{1}([0,1], \mathbb{R})$ satisfying

$$
p \succ 0, \quad q \succ 0 \quad \text { on }\left[0, R_{0}\right] .
$$

Then, there exists $\vartheta_{0} \in(0,1)$ such that for every $d \in \mathbb{R}$ with $d>0$ and for every solution $u_{d}$ of $(2.2)$, there exists $r_{u_{d}} \in\left(0, \min \left\{\rho_{u_{d}}, R_{0}\right\}\right)$ such that

$$
u_{d}\left(r_{u_{d}}\right)=\vartheta_{0} d \quad \text { and } \quad u_{d}(r)>\vartheta_{0} d \quad \forall r \in\left[0, r_{u_{d}}\right) .
$$

Proof. Let us argue by contradiction, assuming that for every $n \in \mathbb{N} \backslash\{1\}$, there exist $d_{n} \in \mathbb{R}$ with $d_{n}>0$ and a solution $u_{d_{n}}$ of $(2.2)$ with $d=d_{n}$ such that

$$
u_{d_{n}}(r)>\left(1-\frac{1}{n}\right) d_{n} \quad \forall r \in\left[0, \min \left\{\rho_{u_{d_{n}}}, R_{0}\right\}\right) .
$$

Let us first show that $\rho_{u_{d_{n}}}>R_{0}$.

From the equation in $(2.2)$, we get that for every $r \in\left[0, \rho_{u_{d_{n}}}\right)$

$$
-r^{N-1} u_{d_{n}}^{\prime}(r)=\int_{0}^{r} s^{N-1}\left|u_{d_{n}}(s)\right|^{\delta-1} u_{d_{n}}(s) q(s) d s+\int_{0}^{r} s^{N-1}\left|u_{d_{n}}(s)\right|^{\gamma-1} u_{d_{n}}(s) p(s) d s .
$$

Hence, taking into account (3.3) and the non-negativeness of $p$ and $q$, it follows that $u_{d_{n}}^{\prime} \leq 0$ on $\left[0, \min \left\{\rho_{u_{d_{n}}}, R_{0}\right\}\right)$ and, consequently,

$$
\left(1-\frac{1}{n}\right) d_{n}<u_{d_{n}}(r) \leq d_{n} \quad \forall r \in\left[0, \min \left\{\rho_{u_{d_{n}}}, R_{0}\right\}\right) .
$$

According to the definitions in (2.12), from (3.4) we immediately deduce that for every $r \in\left[0, \min \left\{\rho_{u_{d_{n}}}, R_{0}\right\}\right)$

$$
-r^{N-1} u_{d_{n}}^{\prime}(r) \leq d_{n}^{\delta} \int_{0}^{r} s^{N-1} q(s) d s+d_{n}^{\gamma} \int_{0}^{r} s^{N-1} p(s) d s \leq\left(d_{n}^{\delta} Q+d_{n}^{\gamma} P\right) \frac{r^{N}}{N}
$$

whence we infer that $u_{d_{n}}^{\prime}(r) \geq-\left(d_{n}^{\delta} Q+d_{n}^{\gamma} P\right) \frac{r}{N}$. In particular,

$$
-\frac{d_{n}^{\delta} Q+d_{n}^{\gamma} P}{N} \leq u_{d_{n}}^{\prime}(r) \leq 0 \quad \forall r \in\left[0, \min \left\{\rho_{u_{d_{n}}}, R_{0}\right\}\right)
$$

which, combined with (3.5), guarantees the uniform boundedness of $u_{d_{n}}$ and $u_{d_{n}}^{\prime}$ on $\left[0, \min \left\{\rho_{u_{d_{n}}}, R_{0}\right\}\right]$. Thus, $u_{d_{n}}$ is globally defined on $\left[0, R_{0}\right]$ and $\rho_{u_{d_{n}}}>R_{0}$. 
From (3.3) and (3.4), we obtain that

$u_{d_{n}}^{\prime}(r) \leq-\left(1-\frac{1}{n}\right)^{\delta} d_{n}^{\delta} \frac{\int_{0}^{r} s^{N-1} q(s) d s}{r^{N-1}}-\left(1-\frac{1}{n}\right)^{\gamma} d_{n}^{\gamma} \frac{\int_{0}^{r} s^{N-1} p(s) d s}{r^{N-1}} \quad \forall r \in\left[0, R_{0}\right]$.

We now integrate and, taking into account the definitions in (2.14) and the inequality (3.3), we finally conclude that

$$
\left(1-\frac{1}{n}\right) d_{n}-d_{n}<u_{d_{n}}\left(R_{0}\right)-d_{n} \leq-\left(1-\frac{1}{n}\right)^{\delta} d_{n}^{\delta} \Psi_{q}\left(R_{0}\right)-\left(1-\frac{1}{n}\right)^{\gamma} d_{n}^{\gamma} \Psi_{p}\left(R_{0}\right),
$$

whence it follows

$$
\left(1-\frac{1}{n}\right)^{\delta} d_{n}^{\delta-1} \Psi_{q}\left(R_{0}\right)+\left(1-\frac{1}{n}\right)^{\gamma} d_{n}^{\gamma-1} \Psi_{p}\left(R_{0}\right)<\frac{1}{n}
$$

Our aim consists in showing that (3.6) leads to a contradiction.

The goal is easily achieved by observing that $\Psi_{p}\left(R_{0}\right)>0$ and $\Psi_{q}\left(R_{0}\right)>0$ from assumption (3.1), and by noticing that, up to a subsequence, $d_{n}$ tends to $d_{0} \in[0,+\infty]$ as $n \rightarrow+\infty$. Passing to the limit on this subsequence, the contradiction follows. In particular, if $d_{0} \in(0,+\infty)$, then (3.6) becomes

$$
d_{0}^{\delta-1} \Psi_{q}\left(R_{0}\right)+d_{0}^{\gamma-1} \Psi_{p}\left(R_{0}\right) \leq 0,
$$

an absurd. It is immediate to verify that also the cases $d_{0}=0$ and $d_{0}=+\infty$ lead to a contradiction. This completes the proof.

Lemma 3.1 could be easily refined into the following.

Lemma 3.2 Consider $p, q \in C^{1}([0,1], \mathbb{R})$ satisfying $(3.1)$, then there exists $\vartheta_{0} \in(0,1)$ such that for every $d \in \mathbb{R} \backslash\{0\}$ and for every solution $u_{d}$ of (2.2), there exists $r_{u_{d}} \in$ $\left(0, \min \left\{\rho_{u_{d}}, R_{0}\right\}\right)$ such that

$$
u_{d}\left(r_{u_{d}}\right)=\vartheta_{0} d \quad \text { and } \quad\left|u_{d}(r)\right|>\vartheta_{0}|d| \quad \forall r \in\left[0, r_{u_{d}}\right) .
$$

By a slight modification of the proofs of Theorem $4-\beta(v)$ and of its Corollay in [35], based on contraction mapping arguments, we can deduce the following uniqueness result

Lemma 3.3 Consider $p, q \in C^{1}([0,1], \mathbb{R})$ satisfying (3.1). Then, for every $d \in \mathbb{R} \backslash\{0\}$, the Cauchy problem (2.2) admits a unique solution $u_{d}$ in $\left[0, r_{u_{d}}\right]$.

In particular, $r_{u_{d}} \equiv r_{d}$ depends only on the initial data.

According to the definitions in (2.14) and to Lemma 3.1 and Lemma 3.2, the following inequalities hold.

Remark 3.4 Consider $p, q \in C^{1}([0,1], \mathbb{R})$ satisfying (3.1). Then, for every $d \in \mathbb{R} \backslash\{0\}$,

$$
\begin{array}{cc}
\vartheta_{0}|d| \leq\left|u_{d}(r)\right| \leq\left|u_{d}(0)\right|=|d| & \forall r \in\left[0, r_{d}\right], \\
\left|u_{d}^{\prime}(r)\right| \leq \frac{|d|^{\delta} \Phi_{q}(r)}{r^{N-1}}+\frac{|d|^{\gamma} \Phi_{p}(r)}{r^{N-1}} & \forall r \in\left[0, r_{d}\right] .
\end{array}
$$


Proof. As a consequence of the relations (3.7) established in the previous lemma combined with

$$
-r^{N-1} u_{d}^{\prime}(r)=\int_{0}^{r} s^{N-1}\left|u_{d}(s)\right|^{\delta-1} u_{d}(s) q(s) d s+\int_{0}^{r} s^{N-1}\left|u_{d}(s)\right|^{\gamma-1} u_{d}(s) p(s) d s,
$$

we immediately deduce that $\left|u_{d}(\cdot)\right|$ is decreasing in $\left[0, r_{d}\right]$. This proves the validity of (3.8). Then, by combining (3.8) with (3.10), the inequalities (3.9) also follow.

In order to perform estimates on the $C^{1}$-norm of the solutions of the initial value problem (2.2), we need some auxiliary lemmas which provide bounds from above on $r_{d}$. Taking into account Lemma 3.2 and the notation introduced in (2.12) and (2.14), we can state the following two results

Lemma 3.5 Consider $p, q \in C^{1}([0,1], \mathbb{R})$ satisfying (3.1). Then, there exists $d^{*}>0$ such that for every $d \in \mathbb{R}$ with $|d|>d^{*}$

$$
r_{d} \geq \Psi_{q}^{-1}\left(|d|^{1-\delta}\left(1-\vartheta_{0}\right)-|d|^{\gamma-\delta} \frac{P}{2 N}\right) .
$$

Proof. We set $d^{*}:=P^{\frac{1}{1-\gamma}}\left[2 N\left(1-\vartheta_{0}\right)\right]^{-\frac{1}{1-\gamma}}$. Recalling that $\gamma<1$, we easily deduce that

$$
|d|^{1-\delta}\left(1-\vartheta_{0}\right)-|d|^{\gamma-\delta} \frac{P}{2 N}=|d|^{1-\delta}\left(\left(1-\vartheta_{0}\right)-|d|^{\gamma-1} \frac{P}{2 N}\right)>0, \text { when }|d|>d^{*} .
$$

Let us first consider $d>d^{*}$. Lemma 3.1 and Remark 3.4 guarantee the existence of $r_{d} \in\left(0, \min \left\{\rho_{u_{d}}, R_{0}\right\}\right)$ such that

$$
u_{d}\left(r_{d}\right)=\vartheta_{0} d \quad \text { and } \quad u_{d}^{\prime}(r) \geq-\frac{d^{\delta} \Phi_{q}(r)}{r^{N-1}}-\frac{d^{\gamma} P}{N} r \quad \forall r \in\left[0, r_{d}\right] .
$$

Hence, by integrating $u_{d}^{\prime}$ in $\left[0, r_{d}\right]$ and recalling the definitions in (2.14), we conclude that

which leads to

$$
\vartheta_{0} d-d=u\left(r_{d}\right)-d \geq-d^{\delta} \Psi_{q}\left(r_{d}\right)-\frac{d^{\gamma} P}{2 N}
$$

$$
\Psi_{q}\left(r_{d}\right) \geq d^{1-\delta}\left(1-\vartheta_{0}\right)-d^{\gamma-\delta} \frac{P}{2 N} .
$$

Due to the inequality (3.12), the relation (3.11) follows.

The case $d<-d^{*}<0$ can be treated in an analogous way.

Lemma 3.6 Consider $p, q \in C^{1}([0,1], \mathbb{R})$ satisfying (3.1). Then, there exists $d_{0} \in$ $\left(0, d^{*}\right)$ such that for every $d \in \mathbb{R} \backslash\{0\}$ with $|d| \leq d_{0}$

$$
r_{d} \geq \Psi_{p}^{-1}\left(|d|^{1-\gamma}\left(1-\vartheta_{0}\right)-|d|^{\delta-\gamma} \frac{Q}{2 N}\right) .
$$


Proof. Recalling that $\gamma<1<\delta$, we easily deduce the existence of $d_{0} \in\left(0, d^{*}\right)$ such that

$$
|d|^{1-\gamma}\left(\left(1-\vartheta_{0}\right)-|d|^{\delta-1} \frac{Q}{2 N}\right)>0, \quad \text { when } 0<|d| \leq d_{0} .
$$

Lemma 3.1 and Remark 3.4 guarantee the existence of $r_{d} \in\left(0, \min \left\{\rho_{u_{d}}, R_{0}\right\}\right)$ such that

$$
u_{d}\left(r_{d}\right)=\vartheta_{0} d \quad \text { and } \quad u_{d}^{\prime}(r) \geq-\frac{d^{\delta} Q}{N} r-\frac{d^{\gamma} \Phi_{p}(r)}{r^{N-1}} \quad \forall r \in\left[0, r_{d}\right] .
$$

Hence, by integrating $u_{d}^{\prime}$ in $\left[0, r_{d}\right]$ and recalling the definitions in $(2.14)$, we conclude that

which leads to

$$
\vartheta_{0} d-d=u\left(r_{d}\right)-d \geq-\frac{d^{\delta} Q}{2 N}-d^{\gamma} \Psi_{p}\left(r_{d}\right)
$$

$$
\Psi_{p}\left(r_{d}\right) \geq d^{1-\gamma}\left(1-\vartheta_{0}\right)-d^{\delta-\gamma} \frac{Q}{2 N} .
$$

Due to the inequality (3.14), the relation (3.13) follows.

The case $d \leq-d_{0}<0$ can be treated in an analogous way.

\section{Global existence results}

This section is devoted to present the global existence result concerning the solutions $u_{d}$ of $(2.2)$ and Cauchy problems with more general initial data. Some remarks about uniqueness conclude the section.

First of all, let us introduce some useful notation. According to (1.7), we denote by $\widetilde{r}_{1}$ the first positive zero of the map $q$. By assumption (1.8) there exist $S_{1} \in\left(R_{1}, \widetilde{r}_{1}\right)$ and $S_{i} \in\left(r_{i-1}, \widetilde{r}_{i}\right)$ with $i \in\{2, \ldots, n\}$ such that

$$
q^{\prime}(r) \leq 0, \quad \forall r \in\left[S_{i}, \widetilde{r}_{i}\right], \quad \forall i \in\{1, \ldots, n\} .
$$

The definition of $S_{i}$ should be further refined according to the behaviour of the weight $p$ in $\widetilde{r}_{i}$. Taking into account (1.7) and our assumption (1.10), we know that $p\left(\widetilde{r}_{i}\right)=0$ implies that $p^{\prime}\left(\widetilde{r}_{i}\right) \leq 0$ in a left neighbourhood of $\widetilde{r}_{i}$ both in the case $\widetilde{r}_{i}=\widetilde{s}_{j_{i}}$ and in the case $\widetilde{r}_{i} \in J_{j_{i}} \backslash \widetilde{s}_{j_{i}}$, for a suitable $j_{i} \in\{1, \ldots, m\}$. Thus, it is not restrictive to choose $S_{i}$ and a positive constant $p_{i}$ satisfying also

$$
\begin{array}{clll}
p(r) \geq p_{i}>0 & \forall r \in\left[S_{i}, \widetilde{r}_{i}\right], & \forall i \in\{1, \ldots, n\}, & \text { if } p\left(\widetilde{r}_{i}\right)>0, \\
p^{\prime}(r) \leq 0 & \forall r \in\left[S_{i}, \widetilde{r}_{i}\right], & \forall i \in\{1, \ldots, n\}, & \text { if } p\left(\widetilde{r}_{i}\right)=0 .
\end{array}
$$

Our first aim consists in showing that the solutions $u_{d}$ of (2.2) are globally defined in $\left[0, \widetilde{r}_{1}\right]$, whenever $d \in \mathbb{R} \backslash\{0\}$.

Lemma 4.1 Consider $p, q \in C^{1}([0,1], \mathbb{R})$ satisfying $(1.2)$, one of the alternatives described in (1.3)-(1.6), (1.7), (1.8) and (1.10). Then, $\rho_{u_{d}}>\widetilde{r}_{1}$ for every solution $u_{d}$ of (2.2) with $d \in \mathbb{R} \backslash\{0\}$. 
Proof. To prove this result we use analogous arguments to the ones developed in Lemma 3.3 of [13]. Let us introduce the function energy $\mathcal{E}_{u_{d}}$ by setting

$$
\mathcal{E}_{u_{d}}(r):=\frac{1}{2} u_{d}^{\prime}(r)^{2}+\frac{q(r)}{\delta+1}\left|u_{d}(r)\right|^{\delta+1}+\frac{p(r)}{\gamma+1}\left|u_{d}(r)\right|^{\gamma+1} \quad \forall r \in\left[0, \rho_{u_{d}}\right) .
$$

Observe that the equation in (2.2) can be equivalently written in the form

$$
u^{\prime \prime}(r)=-|u(r)|^{\delta-1} u(r) q(r)-|u(r)|^{\gamma-1} u(r) p(r)-\frac{N-1}{r} u^{\prime}(r),
$$

whenever $r \in\left(0, \rho_{u_{d}}\right)$. According to (4.5), we obtain that for every $r \in\left(0, \rho_{u_{d}}\right)$

$$
\begin{aligned}
\mathcal{E}_{u_{d}}^{\prime}(r):= & u_{d}^{\prime \prime}(r) u_{d}^{\prime}(r)+\frac{q^{\prime}(r)}{\delta+1}\left|u_{d}(r)\right|^{\delta+1}+q(r)\left|u_{d}(r)\right|^{\delta-1} u_{d}(r) u_{d}^{\prime}(r)+ \\
& +\frac{p^{\prime}(r)}{\gamma+1}\left|u_{d}(r)\right|^{\gamma+1}+p(r)\left|u_{d}(r)\right|^{\gamma-1} u_{d}(r) u_{d}^{\prime}(r)= \\
= & -\frac{N-1}{r} u_{d}^{\prime}(r)^{2}+\frac{q^{\prime}(r)}{\delta+1}\left|u_{d}(r)\right|^{\delta+1}+\frac{p^{\prime}(r)}{\gamma+1}\left|u_{d}(r)\right|^{\gamma+1} .
\end{aligned}
$$

According to (2.13) and (3.8), it immediately follows that

$$
\mathcal{E}_{u_{d}}^{\prime}(r) \leq \frac{Q^{\prime}}{\delta+1}|d|^{\delta+1} \frac{P^{\prime}}{\gamma+1}|d|^{\gamma+1}=: \widetilde{\alpha}_{d} \quad \forall r \in\left[0, r_{d}\right] .
$$

We are now interested in estimating $\mathcal{E}_{u_{d}}^{\prime}$ in the interval $\left(r_{d}, \min \left\{\rho_{u_{d}}, S_{1}\right\}\right)$, where we refer to the beginning of this section for the definition of $S_{1}$.

Setting $A_{u_{d}}:=\left\{r \in\left(r_{d}, \min \left\{\rho_{u_{d}}, S_{1}\right\}\right):\left|u_{d}(r)\right| \leq 1\right\}$ and $B_{u_{d}}:=\left(r_{d}, \min \left\{\rho_{u_{d}}, S_{1}\right\}\right) \backslash A_{u_{d}}$, from (4.6) combined with (2.13) we deduce that

$$
\mathcal{E}_{u_{d}}^{\prime}(r) \leq \frac{Q^{\prime}}{\delta+1}+\frac{P^{\prime}}{\gamma+1}=: \widehat{\alpha}, \quad \forall r \in A_{u_{d}} .
$$

Let us define

$$
q_{d}:=\min _{r \in\left[r_{d}, S_{1}\right]} q(r),
$$

which is positive since $\left[r_{d}, S_{1}\right] \subset\left(0, \widetilde{r}_{1}\right)$, for every $d \neq 0$. Hence, recalling that $\gamma<\delta$ and taking into account $(2.13),(4.4),(4.6)$, we easily obtain the following inequality

$$
\mathcal{E}_{u_{d}}^{\prime}(r) \leq \widetilde{\beta}_{d} \frac{q(r)}{\delta+1}\left|u_{d}(r)\right|^{\delta+1} \leq \widetilde{\beta}_{d} \mathcal{E}_{u_{d}}(r), \quad \forall r \in B_{u_{d}}
$$

where $\widetilde{\beta}_{d}:=\frac{1}{q_{d}}\left(Q^{\prime}+\frac{\delta+1}{\gamma+1} P^{\prime}\right)$.

It remains to provide estimates in the interval $\left[S_{1}, \min \left\{\rho_{u_{d}}, \widetilde{r}_{1}\right\}\right]$, in case $\rho_{u_{d}}>S_{1}$. Note that from (4.1) and (4.6), it follows

$$
\mathcal{E}_{u_{d}}^{\prime}(r) \leq \frac{p^{\prime}(r)}{\gamma+1}\left|u_{d}(r)\right|^{\gamma+1}, \quad \forall r \in\left[S_{1}, \min \left\{\rho_{u_{d}}, \widetilde{r}_{1}\right\}\right] .
$$


Thus, by one of the alternatives in (4.2)-(4.3), we infer the existence of $p_{1}>0$ such that

$$
\mathcal{E}_{u_{d}}^{\prime}(r) \leq \frac{P^{\prime}}{p_{1}} \frac{p(t)}{\gamma+1}\left|u_{d}(r)\right|^{\gamma+1} \leq \frac{P^{\prime}}{p_{1}} \mathcal{E}_{u_{d}}(r), \quad \forall r \in\left[S_{1}, \min \left\{\rho_{u_{d}}, \widetilde{r}_{1}\right\}\right] .
$$

Finally, by combining (4.7)-(4.10), we conclude that

$$
\mathcal{E}_{u_{d}}^{\prime}(r) \leq \alpha_{d}+\beta_{d} \mathcal{E}_{u_{d}}(r) \quad \forall r \in\left[0, \min \left\{\rho_{u_{d}}, \widetilde{r}_{1}\right\}\right),
$$

for suitable positive constants $\alpha_{d}, \beta_{d}$. Now, the Gronwall Lemma ensures that

$$
\mathcal{E}_{u_{d}}(r) \leq\left(\mathcal{E}_{u_{d}}(0)+\frac{\alpha_{d}}{\beta_{d}}\right) e^{\beta_{d} r}-\frac{\alpha_{d}}{\beta_{d}} \leq\left(\frac{q(0)}{\delta+1}|d|^{\delta+1}+\frac{p(0)}{\gamma+1}|d|^{\gamma+1}+\frac{\alpha_{d}}{\beta_{d}}\right) e^{\beta_{d}}=: c_{d},
$$

for every $r \in\left[0, \min \left\{\rho_{u_{d}}, \widetilde{r}_{1}\right\}\right)$. It easily follows that $\left|u_{d}^{\prime}(r)\right| \leq \sqrt{2 c_{d}}$ for every $r \in$ $\left[0, \min \left\{\rho_{u_{d}}, \widetilde{r}_{1}\right\}\right]$, and, consequently,

$$
\left|u_{d}(r)\right| \leq|d|+r \sqrt{2 c_{d}} \leq|d|+\sqrt{2 c_{d}} \quad \forall r \in\left[0, \min \left\{\rho_{u_{d}}, \widetilde{r}_{1}\right\}\right] .
$$

Thus, $u_{d}$ is globally defined on $\left[0, \widetilde{r}_{1}\right]$ and $\rho_{u_{d}}>\widetilde{r}_{1}$, since the solution proceeds further $\widetilde{r}_{1}$ simply by Peano theorem about local existence.

As a second step, we extend the global continuability result to the interval $\left[0, \widetilde{r}_{2}\right]$.

Lemma 4.2 Consider $p, q \in C^{1}([0,1], \mathbb{R})$ satisfying $(1.2)$, one of the alternatives described in (1.3)-(1.6), (1.7), (1.8) and (1.10). Then, each solutions $u_{d}$ of (2.2) with $d \in \mathbb{R} \backslash\{0\}$ is globally defined in $\left[0, \widetilde{r}_{2}\right]$.

Proof. Let $u_{d}$ be a solution of $(2.2)$, with $d \neq 0$. The previous lemma guarantees that $\rho_{u_{d}}>\widetilde{r_{1}}$.

- We first claim that $u_{d}$ is globally defined on $\left[0, r_{1}\right]$.

We are interested in studying the behaviour of the solution in the interval $I_{1}:=\left[\widetilde{r}_{1}, r_{1}\right]$, provided that this set does not degenerate to a single point.

Since $q=q^{\prime}=0$ in $\left(\widetilde{r}_{1}, r_{1}\right)$, the energy expression (4.4) and its derivative reduce to

$$
\mathcal{E}_{u_{d}}(r)=\frac{1}{2} u_{d}^{\prime}(r)^{2}+\frac{p(r)}{\gamma+1}\left|u_{d}(r)\right|^{\gamma+1}, \quad \mathcal{E}_{u_{d}}^{\prime}(r)=-\frac{N-1}{r} u_{d}^{\prime}(r)^{2}+\frac{p^{\prime}(r)}{\gamma+1}\left|u_{d}(r)\right|^{\gamma+1},
$$

for every $r \in\left(\widetilde{r}_{1}, \min \left\{\rho_{u_{d}}, r_{1}\right\}\right)$. Our aim consists in estimating the energy derivative according to the behaviour of the weight $p$. For this reason, we will treat the intervals $\widehat{J}_{j}:=J_{j} \cap\left[\widetilde{r}_{1}, r_{1}\right]$ where $p$ vanishes and, separately, the intervals $\widehat{Z}_{j}:=\left[s_{j}, \widetilde{s}_{j+1}\right] \cap\left[\widetilde{r}_{1}, r_{1}\right]$, in whose interior part $p$ is positive. Denote by $\widehat{Z}_{j}:=\left[s_{j}, \widetilde{s}_{j+1}\right] \cap\left[\widetilde{r}_{1}, r_{1}\right]$ the closure of these intervals in $\left[\widetilde{r}_{1}, r_{1}\right]$. We refer to (1.7) for more details concerning the notation.

We argue iteratively by showing, roughly speaking, that the boundedness of the energy can be extended from an interval to the next one. More precisely, we claim that the existence of a constant $c_{u_{d}}>0$ such that

$$
\mathcal{E}_{u_{d}}(r) \leq c_{u_{d}} \quad \forall r \in\left[0, s_{j}\right] \text { or } \forall r \in\left[0, \widetilde{s}_{j}\right]
$$


implies the existence of $\widetilde{c}_{u_{d}}>0$ such that

$$
\mathcal{E}_{u_{d}}(r) \leq \widehat{c}_{u_{d}} \quad \forall r \in \widehat{Z}_{j} \text { or } \forall r \in \widehat{J}_{j+1}
$$

respectively.

Suppose first the validity of (4.12) in $\left[0, s_{j}\right]$.

As a consequence, $\rho_{u_{d}}>s_{j}$ and $\left|u_{d}\left(s_{j}\right)\right| \leq c_{u_{d}}^{*}$, for a suitable constant $c_{u_{d}}^{*}>0$ depending on $c_{u_{d}}$. Therefore, we can consider $\widehat{r}_{u_{d}} \in\left(s_{j}, \min \left\{\rho_{u_{d}}, \widetilde{s}_{j+1}\right\}\right)$ such that $\left|u_{d}(r)\right| \leq c_{u_{d}}^{*}+1$ for each $r \in\left[s_{j}, \widehat{r}_{u_{d}}\right]$. From (2.13) and (4.11), it immediately follows that

$$
\mathcal{E}_{u_{d}}^{\prime}(r) \leq \frac{P^{\prime}}{\gamma+1}\left(c_{u_{d}}^{*}+1\right)^{\gamma+1}, \quad \forall r \in\left[s_{j}, \widehat{r}_{u_{d}}\right]
$$

Moreover, assumption (1.10) ensures the existence of $\widehat{S}_{j}$ such that $p^{\prime} \leq 0$ in $\left[\widehat{S}_{j}, \widetilde{s}_{j+1}\right]$. Define

$$
p_{u_{d}}:=\min _{r \in\left[\widehat{r}_{u_{d}}, \widehat{S}_{j}\right]} p(r) .
$$

According to (2.13) and (4.11), we conclude that

$$
\mathcal{E}_{u_{d}}^{\prime}(r) \leq \frac{P^{\prime}}{p_{u_{d}}} \frac{p(t)}{\gamma+1}\left|u_{d}(r)\right|^{\gamma+1} \leq \frac{P^{\prime}}{p_{u_{d}}} \mathcal{E}_{u_{d}}(r) \quad \forall r \in\left[\widehat{r}_{u_{d}}, \min \left\{\rho_{u_{d}}, \widetilde{s}_{j+1}\right\}\right],
$$

which, combined with (4.14), allows us to apply the Gronwall Lemma and, consequently, to guarantee the validity of (4.13) in $\widehat{Z}_{j}$. In particular, $\rho_{u_{d}}>\widetilde{s}_{j+1}$.

Suppose now the validity of (4.12) in $\left[0, \widetilde{s}_{j}\right]$.

Recalling that $p=0$ in $\widehat{J}_{j+1}$, we immediately observe that

$$
\mathcal{E}_{u_{d}}^{\prime}(r) \leq \frac{p^{\prime}(r)}{\gamma+1}\left|u_{d}(r)\right|^{\gamma+1} \leq 0, \quad \forall r \in \widehat{J}_{j+1},
$$

from which (4.13) in $\widehat{J}_{j+1}$ follows. In particular, $\rho_{u_{d}}>s_{j+1}$.

Summarizing, we have proved that each solution $u_{d}$ proceeds from one interval to the next one in $I_{1}$. Since, by assumption (1.7) $I_{1}$ can be covered by a finite number of closed intervals of the form $\widehat{J}_{j}$ and $\widehat{Z}_{j}$, according to the conclusions of Lemma 4.1 , we can iteratively deduce the existence of $\bar{c}_{u_{d}}$ such that

$$
\mathcal{E}_{u_{d}}(r) \leq \bar{c}_{u_{d}} \quad \forall r \in\left[0, r_{1}\right] .
$$

This ensures that $\rho_{u_{d}}>r_{1}$ and $u_{d}$ is globally defined on $\left[0, r_{1}\right]$, proving our claim.

- Let us now claim that $u_{d}$ is globally defined on $\left[0, \tilde{r}_{2}\right]$.

The estimates on the energy we are interested in exhibiting can be achieved by means of the previous lemma arguments. Recalling the definition of $S_{2}$ given at the beginning of this section, by (4.15) we are allowed to take $r_{u_{d}} \in\left(r_{1}, \min \left\{\rho_{u_{d}}, S_{2}\right\}\right)$ such that

$$
\left|u_{d}(r)\right| \leq|d|+\sqrt{2 \bar{c}_{u_{d}}}+1, \quad \forall r \in\left[r_{1}, \mathrm{r}_{u_{d}}\right] .
$$


Set $A_{u_{d}}^{2}:=\left\{r \in\left(\mathrm{r}_{u_{d}}, \min \left\{\rho_{u_{d}}, S_{2}\right\}\right):\left|u_{d}(r)\right| \leq 1\right\}$ and $B_{u_{d}}^{2}:=\left(\mathrm{r}_{u_{d}}, \min \left\{\rho_{u_{d}}, S_{2}\right\}\right) \backslash A_{u_{d}}^{2}$. Exactly as in proof of the previous lemma, according to (2.13), (4.7) and (4.16), we deduce that there exists $\alpha_{u_{d}}>0$ such that

$$
\mathcal{E}_{u_{d}}^{\prime}(r) \leq \alpha_{u_{d}}, \quad \forall r \in\left[r_{1}, r_{u_{d}}\right] \cup A_{u_{d}}^{2} .
$$

As in the previous lemma, we define

$$
q_{\mathrm{r}_{u_{d}}}:=\min _{r \in\left[\mathrm{r}_{u_{d}}, S_{2}\right]} q(r)
$$

to guarantees the existence of $\widetilde{\beta}_{\mathbf{r}_{u_{d}}}=\widetilde{\beta}\left(q_{\mathbf{r}_{u_{d}}}\right)$ such that

$$
\mathcal{E}_{u_{d}}^{\prime}(r) \leq \widetilde{\beta}_{\mathbf{r}_{u_{d}}} \frac{q(r)}{\delta+1}\left|u_{d}(r)\right|^{\delta+1} \leq \widetilde{\beta}_{\mathbf{r}_{u_{d}}} \mathcal{E}_{u_{d}}(r), \quad \forall r \in B_{u_{d}}^{2}
$$

The estimates on the set $\left[S_{2}, \min \left\{\rho_{u_{d}}, \widetilde{r}_{2}\right\}\right]$, in case $\rho_{u_{d}}>S_{2}$, follow by assumptions (1.8) and (1.10) and their respective consequences (4.1) and (4.2)-(4.3). In particular, as before we deduce the existence of $p_{2}>0$ such that

$$
\mathcal{E}_{u_{d}}^{\prime}(r) \leq \frac{p^{\prime}(r)}{\gamma+1}\left|u_{d}(r)\right|^{\gamma+1} \leq \frac{P^{\prime}}{p_{2}} \frac{p(t)}{\gamma+1}\left|u_{d}(r)\right|^{\gamma+1} \leq \frac{P^{\prime}}{p_{2}} \mathcal{E}_{u_{d}}(r), \quad \forall r \in\left[S_{2}, \min \left\{\rho_{u_{d}}, \widetilde{r}_{2}\right\}\right] .
$$

Finally, by combining (4.17)-(4.19), we obtain the existence of $\beta_{\mathrm{r}_{u_{d}}}$

$$
\mathcal{E}_{u_{d}}^{\prime}(r) \leq \alpha_{u_{d}}+\beta_{\mathbf{r}_{u_{d}}} \mathcal{E}_{u_{d}}(r) \quad \forall r \in\left[r_{1}, \min \left\{\rho_{u_{d}}, \widetilde{r}_{2}\right\}\right) .
$$

Now, by combining the Gronwall Lemma with the definition of $\bar{c}_{u_{d}}$ in (4.15), we conclude that

$$
\mathcal{E}_{u_{d}}(r) \leq\left(\mathcal{E}_{u_{d}}\left(r_{1}\right)+\frac{\alpha_{u_{d}}}{\beta_{\mathbf{r}_{u_{d}}}}\right) e^{r \beta_{\mathbf{r}_{u_{d}}}}-\frac{\alpha_{u_{d}}}{\beta_{\mathbf{r}_{u_{d}}}} \leq\left(\bar{c}_{u_{d}}+\frac{\alpha_{u_{d}}}{\beta_{\mathbf{r}_{u_{d}}}}\right) e^{\beta_{\mathbf{r}_{u_{d}}}},
$$

for every $r \in\left[r_{1}, \min \left\{\rho_{u_{d}}, \widetilde{r}_{2}\right\}\right)$. This allows us to conclude that $u_{d}$ is globally defined on $\left[0, \widetilde{r}_{2}\right]$ and $\rho_{u_{d}}>\widetilde{r}_{2}$. The thesis is achieved.

The arguments of the previous lemma can be finally adopted to extend the global continuability result to the whole interval $[0,1]$.

Lemma 4.3 Consider $p, q \in C^{1}([0,1], \mathbb{R})$ satisfying (1.2), one of the alternatives described in (1.3)-(1.6), (1.7), (1.8) and (1.10). Then, each solution $u_{d}$ of (2.2) with $d \in \mathbb{R} \backslash\{0\}$ is globally defined in $[0,1]$.

Sketch of the proof. The proof follows by repeating iteratively the arguments of Lemma 4.2 in each interval covering $\left[r_{1}, 1\right]$. We can proceed by estimating the energy in the intervals $I_{i}=\left[\widetilde{r}_{i}, r_{i}\right], i \in\{2, \ldots, n\}$ where $q$ vanishes and, separately, in the intervals in $I_{i}^{c}:=\left[r_{i}, \widetilde{r}_{i+1}\right]$, in whose interior part the weight $q$ is positive.

The intervals $I_{i}, i \geq 2$, can be handled as $I_{1}$ in the proof of Lemma 4.2. According to the 
sign of $p$, we divide each $I_{i}$ in a finite number of sub-intervals, and proceed iteratively. If $p$ vanishes in the sub-interval, then the estimates are immediately achieved since the energy decreases. Otherwise, if $p$ is positive in the interior part of the interval, we proceed further the left extreme of the sub-interval by the classical Peano local existence result. Then, we easily estimate the energy derivative in a sub-interval where $p$ is strictly positive. Finally, when $p$ monotonically decreases to 0 in a left neighborhood of each $s_{j}$ (as assumed in (1.10)) the energy still decresases, and the complete estimates follow.

The remaining intervals $I_{i}^{c}$ can be studied by using the same argument of Lemma 4.1 and of the second claim of Lemma 4.2. In these intervals, the behaviour of $q$ has the priority on the one of $p$. The basic idea consists again in proceeding further $r_{i}$ by the Peano local existence result. Again, we can easily estimate the energy derivative in a sub-interval where $q$ is strictly positive. Finally, when $q$ monotonically decreases to 0 in the left neighborhood of $\widetilde{r}_{i+1}$ defined by $\left[S_{i+1}, \widetilde{r}_{i+1}\right]$ the energy can be easily estimated by (4.1)-(4.3), which take into account also the behaviour of $p$ in such a neighborhood. Since the number of intervals $I_{i}$ and $I_{i}^{c}$ covering $[0,1]$ is finite by assumption (1.7), we achieve the global continuability of the solutions in $[0,1]$ through a finite number of iterative steps.

The global existence results can be extended to the solutions of the Cauchy problem

$$
\left\{\begin{array}{l}
\left(r^{N-1} u^{\prime}(r)\right)^{\prime}+r^{N-1}|u(r)|^{\delta-1} u(r) q(r)+r^{N-1}|u(r)|^{\gamma-1} u(r) p(r)=0, \\
\left(u^{\prime}(\tau), u(\tau)\right)=\mathbf{d} \in \mathbb{R}^{2} .
\end{array}\right.
$$

Lemma 4.4 Fix $\tau \in\left[R_{1}, 1\right], \mathbf{d} \in \mathbb{R}^{2}$, and consider $p, q \in C^{1}([0,1], \mathbb{R})$ satisfying (1.7)(1.10). Then, each solution of (4.20) is globally defined in $\left[R_{1}, 1\right]$.

Sketch of the proof. Arguing exactly as in the previous lemma, we extend each solution of $(4.20)$ to the interval $[\tau, 1]$.

Thus, we only need to prove the global existence of solutions in $\left[R_{1}, \tau\right]$. To achieve this goal, we follow an analogous approach, based on the iterative study of suitable sub-intervals in which both the weights $q$ and $p$ vanish, in which one of the weights is positive and in which at least one of the weights reaches the zero in the extreme point of the sub-interval given by $\widetilde{r}_{i}, r_{i}, \widetilde{s}_{j}, s_{j}$. Again we need to estimate the energy derivative (4.6) to apply the Gronwall Lemma, with the difference that now we are interested in providing lower bounds to the expression in (4.6). Notice that the first addendum in (4.6) is easily estimated by the following

$$
-\frac{N-1}{r} u^{\prime}(r)^{2} \geq-\frac{N-1}{R_{1}} u^{\prime}(r)^{2} \geq-\frac{2(N-1)}{R_{1}} \mathcal{E}_{u}(r) \quad \forall r \in\left[R_{1}, \tau\right] .
$$

On the other hand, the lower bounds on the second and third addendum can be obtained by means of the same techniques developed in the proof of the previous lemmas. In particular, note that the solution immediately extends to the left side of $\widetilde{r}_{i}$ and $\widetilde{s}_{j}$ by the classical Peano local existence result. Observe, moreover, that the lower estimates on the energy derivative in right neighbourhoods of $r_{i}$ and $s_{j}$ should be now based 
on assumptions (1.9) and (1.11), instead of (1.8) and (1.10) employed in the previous lemmas and to extend our solution to $[\tau, 1]$.

We end the Section by presenting some uniqueness results. First of all, taking into account Lemma 3.3 and [35, Theorem $4-\delta($ ii)], we immediately deduce the following.

Remark 4.5 Consider $p, q \in C^{1}([0,1], \mathbb{R})$ satisfying the assumptions of Lemma 4.3. Then, for every $d \in \mathbb{R} \backslash\{0\}$, the Cauchy problem (2.2) admits a unique solution $u_{d}$ in $\left[0, \widetilde{s}_{1}\right)$.

Consider now a solution $u_{d}$ of $(2.2)$ such that $u_{d}(\tau) \neq 0$, for a fixed $\tau \in\left[\widetilde{s}_{1}, 1\right]$. Then, Theorem $4-\beta(\mathrm{v})$ and its Corollary in [35] guarantee that the extension $u_{d}(r)$ remains unique as long as $u_{d}(r) \neq 0$.

Analogously, by [35, Theorem 2] each solution $u_{d}$ of $(2.2)$ with $u_{d}^{\prime}(\tau) \neq 0$ admits a unique extension as long as $u_{d}^{\prime}(r) \neq 0$.

Summarizing these remarks, we can state the following

Remark 4.6 Consider $p, q \in C^{1}([0,1], \mathbb{R})$ satisfying the assumptions of Lemma 4.3. Then, for every $d \in \mathbb{R} \backslash\{0\}$, the solution $u_{d}$ the Cauchy problem (2.2) can lose its uniqueness only in the values $\tau_{0} \in\left[\widetilde{s}_{1}, 1\right]$ in which both $u_{d}$ and $u_{d}^{\prime}$ vanish.

According to $[35$, Theorem $4-\delta(i i)]$, it is easy to verify that $\tau_{0} \in\left\{\widetilde{s}_{1}, \ldots, \widetilde{s}_{m}\right\}$.

Moreover, by the classical continuous dependence results (cf., among others, [20] and [35]), as long as $u_{d}$ preserves its uniqueness, it depends continuously in the $C^{1}$-norm on the initial datum d.

With the same arguments, analogous results for the solutions of (4.20) can be deduced.

Remark 4.7 Consider $p, q \in C^{1}([0,1], \mathbb{R})$ satisfying (1.2), one of the alternatives in (1.3)-(1.6), and (1.7)-(1.10). Then, for every $\mathbf{d} \in \mathbb{R}^{2}$, the solution $u$ of (4.20) with $\tau \in\left[s_{j_{1}}, s_{j_{1}+1}\right) \cup\left(\widetilde{s}_{j_{2}-1}, \widetilde{s}_{j_{2}}\right]$ can lose its uniqueness only in the values $\tau_{0} \in[0,1]$ in which both $u$ and $u^{\prime}$ vanish, which, in turns, implies that $\tau_{0} \in\left\{0, s_{1}, \ldots, s_{j_{1}}, \widetilde{s}_{j_{2}}, \ldots, \widetilde{s}_{m}\right\}$.

In Section 6.2 we show that the solutions corresponding to a suitable range of initial data are uniquely determined.

\section{Small norm solutions of the Cauchy problems in $\left[0, R_{1}\right]$}

This section is devoted to provide upper bounds on the $C^{1}$-norm of the solutions of the Cauchy problem $(2.2)$ in $\left[0, R_{1}\right]$ when the initial data are suitably small.

Lemma 5.1 Consider $p, q \in C^{1}\left(\left[0, R_{1}\right], \mathbb{R}\right)$ satisfying $(1.2)$ and one of the alternatives described in (1.3)-(1.6). Then, for every $\sigma>0$ there exists $d_{\sigma} \in\left(0, d_{0}\right)$ such that for every solution $u_{d}$ of $(2.2)$

$$
\left\|u_{d}\right\|_{1_{\left[0, R_{1}\right]}} \leq \sigma \quad \forall d \in \mathbb{R} \backslash\{0\}:|d| \leq d_{\sigma} .
$$


Proof. Fix $d \in \mathbb{R} \backslash\{0\}$ and consider a solution $u_{d}$ of (2.2).

By Remark 3.4, we observe that for every $\sigma>0$ there exists $\widetilde{d}_{\sigma}>0$ such that

$$
\left\|u_{d}\right\|_{\left.\right|_{\left[0, r_{d}\right]}}<\frac{\sigma}{2} \quad \forall d \in \mathbb{R} \backslash\{0\}:|d| \leq \widetilde{d}_{\sigma} .
$$

Our aim consists now in estimating $\left\|u_{d}\right\|_{1}$ in $\left[r_{d}, R_{1}\right]$.

We analyze separately the different alternatives of our assumptions (1.3)-(1.6).

Assume first the validity of (1.4) and (1.6).

Let us recall the energy definition (4.4) and its derivative calculated in (4.6). According to $(2.5)$ and (2.8), we obtain

$$
\mathcal{E}_{u_{d}}^{\prime}(r) \leq \frac{h+\varepsilon}{r} \frac{q(r)}{\delta+1}\left|u_{d}(r)\right|^{\delta+1}+\frac{k+\varepsilon}{r} \frac{p(r)}{\gamma+1}\left|u_{d}(r)\right|^{\gamma+1} \quad \forall r \in\left[0, R_{1}\right],
$$

which implies that

$$
\mathcal{E}_{u_{d}}^{\prime}(r) \leq \frac{\max \{h, k\}+\varepsilon}{r} \mathcal{E}_{u_{d}}(r) \quad \forall r \in\left[r_{d}, R_{1}\right] \subset\left[0, R_{1}\right] .
$$

Define

$$
c:=\max \{h, k\}+\varepsilon .
$$

Then, by applying the Gronwall Lemma, we deduce that

$$
\mathcal{E}_{u_{d}}(r) \leq \mathcal{E}_{u_{d}}\left(r_{d}\right) e^{\int_{r_{d}}^{r} \frac{c d s}{s}}=\mathcal{E}_{u_{d}}\left(r_{d}\right) e^{c\left(\log r-\log r_{d}\right)}=\mathcal{E}_{u_{d}}\left(r_{d}\right) \frac{r^{c}}{r_{d}^{c}},
$$

for every $r \in\left[r_{d}, R_{1}\right]$. Taking into account Lemma 3.2 and Remark 3.4, we infer that for every $r \in\left[r_{d}, R_{1}\right]$

$$
\mathcal{E}_{u_{d}}(r) \leq \frac{1}{2} \frac{\left(|d|^{\delta} \Phi_{q}\left(r_{d}\right)+|d|^{\gamma} \Phi_{p}\left(r_{d}\right)\right)^{2}}{r_{d}^{(2 N-2)} r_{d}^{c}}+\frac{q\left(r_{d}\right)}{\delta+1} \frac{|d|^{\delta+1}}{r_{d}^{c}}+\frac{p\left(r_{d}\right)}{\gamma+1} \frac{|d|^{\gamma+1}}{r_{d}^{c}} .
$$

According to Remark 2.1, to assumptions (1.4), (1.6), respectively, and to their straightforward consequences $(2.16),(2.17)$, it is not restrictive to choose $\varepsilon>0$ and $R_{1} \in(0,1)$ satisfying the further conditions

$$
\begin{array}{lll}
q(r) \leq(\beta+\varepsilon) r^{h}, & \Phi_{q}(r) \leq \frac{\beta+\varepsilon}{h+N} r^{h+N}, & \forall r \in\left(0, R_{1}\right] \\
p(r) \leq(\alpha+\varepsilon) r^{k}, & \Phi_{p}(r) \leq \frac{\alpha+\varepsilon}{k+N} r^{k+N} & \forall r \in\left(0, R_{1}\right] .
\end{array}
$$

Thus, we deduce the existence of five positive constants $c_{i}=c_{i}(\alpha, \beta, h, k, \varepsilon, \delta, \gamma, N)$, $i \in\{1, \ldots, 5\}$ such that for every $r \in\left[r_{d}, R_{1}\right]$

$\mathcal{E}_{u_{d}}(r) \leq c_{1} r_{d}^{2 h-c+2}|d|^{2 \delta}+c_{2} r_{d}^{2 k-c+2}|d|^{2 \gamma}+c_{3} r_{d}^{h+k-c+2}|d|^{\delta+\gamma}+c_{4} r_{d}^{h-c}|d|^{\delta+1}+c_{5} r_{d}^{k-c}|d|^{\gamma+1}$. 
We have exhibited an upper bound on $\mathcal{E}_{u_{d}}$ which depends on the initial data, but does not depend on the particular solution $u_{d}$. Our aim consists in showing that this upper bound tends to zero as $d \rightarrow 0$.

To this purpose, we need to establish a relation between $r_{d}$ and $d$. Let us first recall that Lemma 3.6 ensures that

$$
r_{d} \geq \Psi_{p}^{-1}\left(|d|^{1-\gamma}\left(1-\vartheta_{0}\right)-|d|^{\delta-\gamma} \frac{Q}{2 N}\right) \quad \forall d \in \mathbb{R} \backslash\{0\}:|d| \leq d_{0} .
$$

Combining this estimate with the relation

$$
\Psi_{p}^{-1}(r) \sim\left(\frac{(k+2)(k+N)}{\alpha}\right)^{\frac{1}{k+2}} r^{\frac{1}{k+2}} \quad \text { when } r \rightarrow 0^{+},
$$

obtained by (2.17), we deduce that there exist $\tilde{c}=\tilde{c}\left(k, N, \alpha, \vartheta_{0}, Q, \delta, \gamma\right)>0$ and $\tilde{d}_{0}=$ $\tilde{d}_{0}\left(k, N, \alpha, \vartheta_{0}, Q, \delta, \gamma\right) \in\left(0, d_{0}\right)$ such that

$$
\frac{1}{r_{d}} \leq \frac{\tilde{c}}{|d|^{\frac{1-\gamma}{k+2}}} \quad \forall d \in \mathbb{R} \backslash\{0\}:|d| \leq \tilde{d}_{0}
$$

This relation allows us to easily estimate the upper bound in (5.7) when $h \leq k$. Let us first concentrate on this easiest case.

- Assume that $h \leq k$.

Recalling the definition of $c$ in (5.2) and the fact that $r_{d}<R_{1} \leq 1$, it is immediate to verify that the third addendum in (5.7) tends to zero as $d \rightarrow 0$.

Since $h \leq k$, we know that $c=k+\varepsilon$. Therefore, also the second addendum in (5.7) tends to zero as $d \rightarrow 0$.

As far as the fourth and fifth addendum are concerned, from (5.8) it follows

$$
\frac{c_{4}|d|^{\delta+1}}{r_{d}^{k-h+\varepsilon}}+\frac{c_{5}|d|^{\gamma+1}}{r_{d}^{\varepsilon}} \leq \tilde{c}_{4} \frac{|d|^{\delta+1}}{|d|^{\frac{(1-\gamma)(k-h+\varepsilon)}{k+2}}}+\tilde{c}_{5} \frac{|d|^{\gamma+1}}{|d|^{\frac{\varepsilon(1-\gamma)}{k+2}}} \rightarrow 0 \quad \text { as } d \rightarrow 0,
$$

where $\tilde{c}_{4}, \tilde{c}_{5}$ are suitable positive constants.

As for the first addendum, we immediately note that it converges to zero when $2 h-k-$ $\varepsilon+2 \geq 0$. Otherwise, according to (5.8), we infer that

$$
\frac{c_{1}|d|^{2 \delta}}{r_{d}^{k+\varepsilon-2 h-2}} \leq \tilde{c}_{1} \frac{|d|^{2 \delta}}{|d|^{\frac{(1-\gamma)(k+-2 h-2)}{k+2}}} \rightarrow 0 \quad \text { as } d \rightarrow 0,
$$

where $\tilde{c}_{1}$ is a suitable positive constant. Our goal is achieved.

- Assume now that $h>k$.

In this case, we cannot use relation (5.8) to prove that the second and the fifth addenda in (5.7) tend to zero as $d \rightarrow 0$. To complete the proof, some more steps are required. 
First of all, from (1.4) and (1.6), it is not restrictive to choose $\varepsilon \in(0,1), R_{1} \in(0,1)$ in Remark 2.1 such that

$$
\begin{aligned}
& q^{\prime}(r) \leq \frac{(\beta+\varepsilon) h}{\alpha} \frac{r^{h-1}}{r^{k}} p(r), \quad \forall r \in\left(0, R_{1}\right], \\
& h>k+2 \varepsilon, \quad R_{1}^{h-k} \leq \frac{\alpha \varepsilon(\delta+1)}{(\beta+\varepsilon) h(\gamma+1)} .
\end{aligned}
$$

Moreover, without loss of generality, we can choose $\sigma<1$ and $\widetilde{d}_{\sigma} \in\left(0, \tilde{d}_{0}\right)$ (cf. (5.1) and (5.8) for the definitions of $\widetilde{d}_{\sigma}$ and $\tilde{d}_{0}$, respectively).

In the spirit of Lemma 2.3 in [9] and according to (5.1), we consider the maximal value $R_{d} \in\left(r_{d}, R_{1}\right]$ in which the following inequalities are satisfied

$$
\left|u_{d}(r)\right| \leq \frac{\sigma}{2}, \quad\left|u_{d}^{\prime}(r)\right| \leq \frac{\sigma}{2} \quad \forall r \in\left[0, R_{d}\right], \quad 0<|d| \leq \widetilde{d}_{\sigma} .
$$

From (4.6), (5.9) and (2.8), we deduce that for every $r \in\left[r_{d}, R_{d}\right]$

$$
\begin{aligned}
\mathcal{E}_{u_{d}}^{\prime}(r) & \leq \frac{q^{\prime}(r)\left|u_{d}(r)\right|^{\delta+1}}{\delta+1}+\frac{p^{\prime}(r)\left|u_{d}(r)\right|^{\gamma+1}}{\gamma+1} \leq \\
& \leq\left(\frac{(\beta+\varepsilon) h r^{h-k}}{\alpha r} \frac{\gamma+1}{\delta+1}+\frac{k+\varepsilon}{r}\right) \frac{p(r)\left|u_{d}(r)\right|^{\gamma+1}}{\gamma+1}
\end{aligned}
$$

Moreover, (5.10) guarantees that

$$
\frac{(\beta+\varepsilon) h r^{h-k}}{\alpha r} \frac{\gamma+1}{\delta+1} \leq \frac{\varepsilon}{r} \quad \forall r \in\left(0, R_{1}\right] .
$$

As a consequence,

$$
\mathcal{E}_{u_{d}}^{\prime}(r) \leq \frac{k+2 \varepsilon}{r} \frac{p(r)\left|u_{d}(r)\right|^{\gamma+1}}{\gamma+1} \leq \frac{k+2 \varepsilon}{r} \mathcal{E}_{u_{d}}(r) \quad \forall r \in\left[r_{d}, R_{d}\right]
$$

As before, we can now get (5.3), (5.4) and (5.7) for every $r \in\left[r_{d}, R_{d}\right]$, where $c:=k+2 \varepsilon$. In particular, for every $r \in\left[r_{d}, R_{d}\right]$, the energy $\mathcal{E}_{u_{d}}(r)$ is less than or equal to $c_{1} r_{d}^{2 h-k+2-2 \varepsilon}|d|^{2 \delta}+c_{2} r_{d}^{k+2-2 \varepsilon}|d|^{2 \gamma}+c_{3} r_{d}^{h+2-2 \varepsilon}|d|^{\delta+\gamma}+c_{4} r_{d}^{h-k-2 \varepsilon}|d|^{\delta+1}+c_{5} r_{d}^{-2 \varepsilon}|d|^{\gamma+1}$.

Taking into account (5.8), (5.10) and recalling that $R_{d} \leq R_{1}<1$, we infer the existence of $\bar{c}_{5}>0$ such that for every $d \in \mathbb{R} \backslash\{0\}$, with $|d| \leq \widetilde{d}_{\sigma}$

$$
\mathcal{E}_{u_{d}}(r) \leq c_{1}|d|^{2 \delta}+c_{2}|d|^{2 \gamma}+c_{3}|d|^{\delta+\gamma}+c_{4}|d|^{\delta+1}+\bar{c}_{5} \frac{|d|^{\gamma+1}}{|d|^{\frac{2 \varepsilon(1-\gamma)}{k+2}}} \rightarrow 0 \quad \text { as } d \rightarrow 0 .
$$

This guarantees the existence of $d_{\sigma} \in\left(0, \widetilde{d}_{\sigma}\right)$ such that

$$
\left|u_{d}(r)\right|<\frac{\sigma}{2}, \quad\left|u_{d}^{\prime}(r)\right|<\frac{\sigma}{2} \quad \forall r \in\left[0, R_{d}\right], \quad 0<|d| \leq d_{\sigma} .
$$


By the maximality of the choice of $R_{d}$ satisfying (5.11), we conclude that $R_{d}=R_{1}$ for every $d \in \mathbb{R} \backslash\{0\}$ with $|d| \leq d_{\sigma}$, from which it follows

$$
\left\|u_{d}\right\|_{1_{\left[0, R_{1}\right]}} \leq \sigma \quad \forall d \in \mathbb{R} \backslash\{0\}:|d| \leq d_{\sigma} .
$$

This completes the proof of the lemma under the assumptions (1.4) and (1.6).

\section{Assume secondly the validity of (1.3) and (1.6).}

Taking into account the energy derivative expression (4.6), from (2.4), (2.8) and (2.13), we obtain

$$
\mathcal{E}_{u_{d}}^{\prime}(r) \leq \frac{Q^{\prime}}{q_{0}} \frac{q(r)}{\delta+1}\left|u_{d}(r)\right|^{\delta+1}+\frac{k+\varepsilon}{r} \frac{p(r)}{\gamma+1}\left|u_{d}(r)\right|^{\gamma+1} \quad \forall r \in\left[0, R_{1}\right] .
$$

According to assumption (1.3), it is not restrictive to choose $\varepsilon>0, q_{0}>0$ and $R_{1} \in(0,1)$ in Remark 2.1 satisfying also the following inequality

$$
\frac{Q^{\prime}}{q_{0}} \leq \frac{k+\varepsilon}{r}, \quad \forall r \in\left(0, R_{1}\right] .
$$

Hence, recalling the energy definition (4.4) we infer that

$$
\mathcal{E}_{u_{d}}^{\prime}(r) \leq \frac{k+\varepsilon}{r} \mathcal{E}_{u_{d}}(r) \quad \forall r \in\left[r_{d}, R_{1}\right] \subset\left(0, R_{1}\right] .
$$

Exactly as in the previous case, by Gronwall Lemma we get (5.4) with $c:=k+\varepsilon$.

According to condition (2.15), it is not rescrictive to require the positive costants $\varepsilon, R_{1}$ in Remark 2.1 to satisfy the further inequality

$$
\Phi_{q}(r) \leq \frac{q(0)+\varepsilon}{N} r^{N}, \quad \forall r \in\left(0, R_{1}\right] .
$$

By combining (5.4) with (5.6) and (5.13), we deduce the existence of five positive constants $\bar{c}_{i}=\bar{c}_{i}(\alpha, k, \varepsilon, \delta, \gamma, N, Q, q(0)), i \in\{1, \ldots, 5\}$ such that for every $r \in\left[r_{d}, R_{1}\right]$

$\mathcal{E}_{u_{d}}(r) \leq \bar{c}_{1} r_{d}^{2-k-\varepsilon}|d|^{2 \delta}+\bar{c}_{2} r_{d}^{k-\varepsilon+2}|d|^{2 \gamma}+\bar{c}_{3} r_{d}^{-\varepsilon+2}|d|^{\delta+\gamma}+\bar{c}_{4} r_{d}^{-k-\varepsilon}|d|^{\delta+1}+\bar{c}_{5} r_{d}^{-\varepsilon}|d|^{\gamma+1}$.

As before, we immediately notice that both the second and the third addendum tend to zero as $d \rightarrow 0$. The estimates of the other addenda are based on (5.8). In particular, from (5.8) we deduce the existence of two positive constants $\tilde{c}_{4}, \tilde{c}_{5}$ such that

$$
\frac{\bar{c}_{4}|d|^{\delta+1}}{r_{d}^{k+\varepsilon}}+\frac{\bar{c}_{5}|d|^{\gamma+1}}{r_{d}^{\varepsilon}} \leq \tilde{c}_{4} \frac{|d|^{\delta+1}}{|d|^{\frac{(1-\gamma)(k+\varepsilon)}{k+2}}}+\tilde{c}_{5} \frac{|d|^{\gamma+1}}{|d|^{\frac{\varepsilon(1-\gamma)}{k+2}}} \rightarrow 0 \quad \text { as } d \rightarrow 0 .
$$

If $k+\varepsilon \leq 2$, it is immediate to note that the first addendum converges to zero. Otherwise, (5.8) ensures the existence of $\tilde{c}_{1}>0$ such that

$$
\frac{\bar{c}_{1}|d|^{2 \delta}}{r_{d}^{k+\varepsilon-2}} \leq \tilde{c}_{1} \frac{|d|^{2 \delta}}{|d|^{\frac{(1-\gamma)(k+\varepsilon-2)}{k+2}}} \rightarrow 0 \quad \text { as } d \rightarrow 0 .
$$


This completes the proof of the lemma under the assumptions (1.3) and (1.6).

Finally, assume the validity of (1.5) combined with the assumption $q \geq 0$. Define

$$
F(r, x):=\frac{q(r)}{\delta+1}|x|^{\delta+1}+\frac{p(r)}{\gamma+1}|x|^{\gamma+1}, \quad \forall(r, x) \in\left[0, R_{1}\right] \times \mathbb{R} .
$$

According to $(2.7)$, observe that for every $(r, x) \in\left[0, R_{1}\right] \times[-1,1]$

$$
\left|\frac{\partial F(r, x)}{\partial r}\right| \leq \frac{\left|q^{\prime}(r)\right|}{\delta+1}|x|^{\delta+1}+\frac{\left|p^{\prime}(r)\right|}{\gamma+1}|x|^{\gamma+1} \leq \varphi(r) \frac{p(r)}{\gamma+1}|x|^{\gamma+1} \leq \varphi(r) F(r, x),
$$

where $\varphi(r):=\left(\frac{\left|q^{\prime}(r)\right|(\gamma+1)}{p_{0}(\delta+1)}+\frac{\left|p^{\prime}(r)\right|}{p_{0}}\right)$. The goal follows by a straightforward application of Lemma 2.3 in [9].

\section{Elastic property}

The aim of this section consists in showing some qualitative properties of the solutions to (2.2) with large initial data. In particular, we are concerned with the validity of Elastic property, which ensures arbitrarily large norms of the solutions to (2.2) for suitably large values of $|d|$.

\subsection{Elastic property in the ball $|x| \leq R_{1}$}

Let us focus our attention on the Elastic property in the interval $\left[0, R_{1}\right]$.

Proposition 6.1 Under the assumptions of Theorem 1.1, for every $\mu>0, \eta>0$ and $L_{1}>0$ there exists $L_{2}>0$ such that for every $d \in \mathbb{R}$ with $|d| \geq L_{2}$

$$
\mu u_{d}(r)^{2}+\eta u_{d}^{\prime}(r)^{2} \geq L_{1}, \quad \forall r \in\left[0, R_{1}\right] .
$$

Due to Lemma 3.2 , we can easily verify the validity of the Elastic property in $\left[0, r_{d}\right)$, whenever $d$ is chosen large enough. Indeed, for every $\mu>0$ and $\eta>0$,

$$
\mu u_{d}(r)^{2}+\eta u_{d}^{\prime}(r)^{2} \geq \mu u_{d}(r)^{2} \geq \mu \vartheta_{0}^{2} d^{2}, \quad \forall r \in\left[0, r_{d}\right],
$$

whence (6.1) restricted to the interval $\left[0, r_{d}\right]$ immediately follows.

Taking into account (2.10) and (2.11), we now take a positive constant $a$ satisfying

$$
\begin{gathered}
\frac{N-2}{2}<a<\frac{N-\varepsilon}{\delta+1}<\frac{N-\varepsilon}{\gamma+1} \quad \text { under assumption (1.12) } \\
\frac{N-2}{2}<a<\min \left\{\frac{N+h-\varepsilon}{\delta+1}, \frac{N-\varepsilon}{\gamma+1}\right\} \quad \text { under assumption (1.13). }
\end{gathered}
$$


Hence, we can deduce the existence of a suitably small $\hat{\varepsilon}=\hat{\varepsilon}(N, \delta, \gamma, \varepsilon, q)>0$ satisfying

$$
\begin{array}{cc}
\frac{N-\varepsilon}{\delta+1}-a>\hat{\varepsilon} & \text { under assumption (1.12), } \\
\frac{N+h-\varepsilon}{\delta+1}-a>\hat{\varepsilon} & \text { under assumption (1.13). }
\end{array}
$$

According to this choice and to Remark 4.5 ensuring the uniqueness of $u_{d}$ in $\left[0, R_{1}\right] \subset$ $\left[0, \widetilde{s}_{1}\right)$, we can set for every $r \in\left[0, R_{1}\right]$

$\mathrm{E}_{d}(r):=\frac{1}{2} r^{N} u_{d}^{\prime}(r)^{2}+r^{N} \frac{q(r)}{\delta+1}\left|u_{d}(r)\right|^{\delta+1}+r^{N} \frac{p(r)}{\gamma+1}\left|u_{d}(r)\right|^{\gamma+1}+a r^{N-1} u_{d}^{\prime}(r) u_{d}(r)$.

Proposition 6.2 Assume (1.2) and one of the alternatives (1.5)-(1.6). Moreover, suppose either (1.3) and (1.12) or (1.4) and (1.13). Then, there exists a positive constant $C:=\hat{\varepsilon} \vartheta_{0}^{\delta+1}$, such that for every $d \in \mathbb{R}$ with $|d| \geq d^{*}$

$$
\mathrm{E}_{d}(r) \geq C|d|^{\delta+1} \Phi_{q}\left(\Psi_{q}^{-1}\left(|d|^{1-\delta}\left(1-\vartheta_{0}\right)-|d|^{\gamma-\delta} \frac{P}{2 N}\right)\right) \quad \forall r \in\left[r_{d}, R_{1}\right] .
$$

Proof. From (4.5), it is immediate to verify that for every $r \in\left[0, R_{1}\right]$

$$
\begin{aligned}
\mathrm{E}_{d}^{\prime}(r) & =r^{N-1} u_{d}^{\prime}(r)^{2}\left(a-\frac{N-2}{2}\right)+r^{N-1}\left|u_{d}(r)\right|^{\delta+1}\left(r \frac{q^{\prime}(r)}{\delta+1}+\frac{N q(r)}{\delta+1}-a q(r)\right)+ \\
& +r^{N-1}\left|u_{d}(r)\right|^{\gamma+1}\left(r \frac{p^{\prime}(r)}{\gamma+1}+\frac{N p(r)}{\gamma+1}-a p(r)\right) .
\end{aligned}
$$

By both (6.3) and (6.4), we deduce that

$$
\begin{aligned}
\mathrm{E}_{d}^{\prime}(r) & \geq r^{N-1}\left|u_{d}(r)\right|^{\delta+1}\left(\frac{r q^{\prime}(r)+N q(r)}{\delta+1}-a q(r)\right)+ \\
& +r^{N-1}\left|u_{d}(r)\right|^{\gamma+1}\left(\frac{r p^{\prime}(r)+N p(r)}{\gamma+1}-a p(r)\right) .
\end{aligned}
$$

Moreover, according to (2.6) and (2.8) (consequence of our assumptions (1.5) and (1.6), respectively), it follows that for every $r \in\left[0, R_{1}\right]$

$$
\mathrm{E}_{d}^{\prime}(r) \geq r^{N-1}\left|u_{d}(r)\right|^{\delta+1}\left(\frac{r q^{\prime}(r)+N q(r)}{\delta+1}-a q(r)\right)+r^{N-1}\left|u_{d}(r)\right|^{\gamma+1} p(r)\left(\frac{N-\varepsilon}{\gamma+1}-a\right) .
$$

Taking into account (2.3), (2.5) and (6.5)-(6.6), we immediately obtain

$$
\begin{gathered}
\left(\frac{r q^{\prime}(r)+N q(r)}{\delta+1}-a q(r)\right) \geq q(r)\left(\frac{N-\varepsilon}{\delta+1}-a\right) \geq q(t) \hat{\varepsilon}, \quad \text { if (1.12) holds, } \\
\left(\frac{r q^{\prime}(r)+N q(r)}{\delta+1}-a q(r)\right) \geq q(r)\left(\frac{N+h-\varepsilon}{\delta+1}-a\right) \geq q(t) \hat{\varepsilon}, \quad \text { if (1.13) holds. }
\end{gathered}
$$


Thus, using one of (6.3)-(6.4) we infer from (6.10) that

$$
\mathrm{E}_{d}^{\prime}(r) \geq r^{N-1}\left|u_{d}(r)\right|^{\delta+1} q(t) \hat{\varepsilon} \quad \forall r \in\left[0, R_{1}\right] .
$$

Our aim consists in proving the validity of (6.7). According to (6.11), observe that

$$
\mathrm{E}_{d}(r)=\int_{0}^{r} \mathrm{E}_{d}^{\prime}(s) d s=\int_{0}^{r_{d}} \mathrm{E}_{d}^{\prime}(s) d s+\int_{r_{d}}^{r} \mathrm{E}_{d}^{\prime}(s) d s \geq \int_{0}^{r_{d}} \mathrm{E}_{d}^{\prime}(s) d s, \quad \forall r \in\left[r_{d}, R_{1}\right] .
$$

By combining (3.8) with (6.11) and recalling the definition of $\Phi_{q}$ in (2.14), it follows that for every $r \in\left[r_{d}, R_{1}\right]$

$$
\mathrm{E}_{d}(r) \geq \hat{\varepsilon} \int_{0}^{r_{d}} s^{N-1}\left|u_{d}(s)\right|^{\delta+1} q(s) d s \geq \hat{\varepsilon} \vartheta_{0}^{\delta+1}|d|^{\delta+1} \Phi_{q}\left(r_{d}\right) .
$$

Recalling now that $\Phi_{q}$ is nondecreasing, from Lemma 3.5 we infer that for every $d \in \mathbb{R}$ with $|d| \geq d^{*}$ and for every $r \in\left[r_{d}, R_{1}\right]$

$$
\mathrm{E}_{d}(r) \geq \hat{\varepsilon} \vartheta_{0}^{\delta+1}|d|^{\delta+1} \Phi_{q}\left(\Psi_{q}^{-1}\left(|d|^{1-\delta}\left(1-\vartheta_{0}\right)-|d|^{\gamma-\delta} \frac{P}{2 N}\right)\right),
$$

whence the thesis follows.

Proposition 6.3 Assume (1.2) and one of the alternatives (1.5)-(1.6). Moreover, suppose either (1.3) and (1.12) or (1.4) and (1.13). Then, for every $K>0$ there exists $d_{K} \geq d^{*}$ such that for every $d \in \mathbb{R}$ with $|d|>d_{K}$

$$
\mathrm{E}_{d}(r) \geq K, \quad \forall r \in\left[r_{d}, R_{1}\right] .
$$

Proof. We analyze separately the two alternatives of our assumptions.

Assume first the validity of (1.3) and (1.12). Condition (2.15) guarantees that

$$
\Phi_{q}\left(\Psi_{q}^{-1}(r)\right) \sim N^{\frac{N-2}{2}} q(0)^{-\frac{N-2}{2}} 2^{\frac{N}{2}} r^{\frac{N}{2}}=: \chi r^{\frac{N}{2}}, \quad \text { as } r \rightarrow 0^{+} .
$$

As a consequence, there exists $\rho_{0}>0$ such that

$$
\Phi_{q}\left(\Psi_{q}^{-1}(r)\right) \geq(1-\varepsilon) \chi r^{\frac{N}{2}} \quad \forall r \in\left[0, \rho_{0}\right] .
$$

Recalling (3.12) and taking into account that $\gamma<1<\delta$, we infer the existence of $\hat{d}^{*}>d^{*}$ such that for every $d \in \mathbb{R}$ with $|d| \geq \hat{d}^{*}$

$$
0 \leq|d|^{1-\delta}\left(1-\vartheta_{0}\right)-|d|^{\gamma-\delta} \frac{P}{2 N} \leq \rho_{0}
$$

Hence, Proposition 6.2 ensures that for every $d \in \mathbb{R}$ with $|d| \geq \hat{d}^{*}$

$$
\mathrm{E}_{d}(r) \geq C|d|^{\delta+1}(1-\varepsilon) \chi\left(|d|^{1-\delta}\left(1-\vartheta_{0}\right)-|d|^{\gamma-\delta} \frac{P}{2 N}\right)^{\frac{N}{2}}, \quad \forall r \in\left[r_{d}, R_{1}\right],
$$


which leads to

$$
\mathrm{E}_{d}(r) \geq \widetilde{C}|d|^{\delta+1}|d|^{\frac{(1-\delta) N}{2}}\left(\left(1-\vartheta_{0}\right)-|d|^{\gamma-1} \frac{P}{2 N}\right)^{\frac{N}{2}}, \quad \forall r \in\left[r_{d}, R_{1}\right],
$$

where we set $\widetilde{C}:=C(1-\varepsilon) \chi>0$. Passing to the limit as $|d| \rightarrow+\infty$ and taking into account that (1.12) can be equivalently written in the form

$$
\delta+1+\frac{(1-\delta) N}{2}>0,
$$

we can deduce the existence of $d_{K} \geq \hat{d}^{*}$ such that for every $d \in \mathbb{R}$ with $|d| \geq d_{K}$

$$
\mathrm{E}_{d}(r) \geq K, \quad \forall r \in\left[r_{d}, R_{1}\right] .
$$

Assume now the validity of (1.4) and (1.13). The condition (2.16) guarantees that

$$
\Phi_{q}\left(\Psi_{q}^{-1}(r)\right) \sim(h+N)^{\frac{N-2}{h+2}} \beta^{-\frac{N-2}{h+2}}(h+2)^{\frac{h+N}{h+2}} r^{\frac{h+N}{h+2}}:=\chi r^{\frac{h+N}{h+2}} \quad \text { as } r \rightarrow 0^{+} .
$$

As a consequence, there exists $\rho_{0}>0$ such that

$$
\Phi_{q}\left(\Psi_{q}^{-1}(r)\right) \geq(1-\varepsilon) \chi r^{\frac{h+N}{h+2}} \quad \forall r \in\left[0, \rho_{0}\right] .
$$

As before, by (3.12) we infer the existence of $\hat{d}^{*}>d^{*}$ such that (6.12) holds for every $d \in \mathbb{R}$ with $|d| \geq \hat{d}^{*}$. By combining Proposition 6.2 with (6.12), we deduce that for every $d \in \mathbb{R}$ with $|d| \geq \hat{d}^{*}$

$$
\mathrm{E}_{d}(r) \geq C|d|^{\delta+1}(1-\varepsilon) \chi\left(|d|^{1-\delta}\left(1-\vartheta_{0}\right)-|d|^{\gamma-\delta} \frac{P}{2 N}\right)^{\frac{h+N}{h+2}}, \quad \forall r \in\left[r_{d}, R_{1}\right],
$$

which leads to

$$
\mathrm{E}_{d}(r) \geq \widetilde{C}|d|^{\delta+1}|d|^{\frac{(1-\delta)(h+N)}{(h+2)}}\left(\left(1-\vartheta_{0}\right)-|d|^{\gamma-1} \frac{P}{2 N}\right)^{\frac{h+N}{h+2}}, \quad \forall r \in\left[r_{d}, R_{1}\right],
$$

where $\widetilde{C}:=C(1-\varepsilon) \chi$. Passing to the limit as $|d| \rightarrow+\infty$ and taking into account that (1.13) can be equivalently written in the form

$$
\delta+1+\frac{(1-\delta)(h+N)}{(h+2)}>0,
$$

we can conclude that there exists $d_{K} \geq \hat{d}^{*}$ such that for every $d \in \mathbb{R}$ with $|d| \geq d_{K}$

$$
\mathrm{E}_{d}(r) \geq K, \quad \forall r \in\left[r_{d}, R_{1}\right] .
$$

This completes the proof.

Recalling the definition of $\mathrm{E}_{d}$, we can easily infer from Proposition 6.3 that for every $\mu>0, \eta>0$ and $L_{1}>0$ there exists $L_{2}>0$ such that for every $d \in \mathbb{R}$ with $|d| \geq L_{2}$

$$
\mu u_{d}(r)^{2}+\eta u_{d}^{\prime}(r)^{2} \geq L_{1}, \quad \forall r \in\left[r_{d}, R_{1}\right] .
$$

Taking into account (6.2), we can conclude the proof of Proposition 6.1. 


\subsection{Elastic property in the annulus $R_{1} \leq|x| \leq 1$}

We are interested in proving that there exists $\mathcal{L}>0$ such that, for every $d \in \mathbb{R}$ with $|d| \geq \mathcal{L}, u_{d}$ can be uniquely extended to the whole interval $[0,1]$.

According to Remark 4.6, this result is obviously ensured by the validity of the Elastic property in the whole interval $[0,1]$, on which we are going to devote our attention.

First of all, we show that the interval of validity of the Elastic property could be extended as long as the solutions of the Cauchy problems remain unique. More precisely, setting $\widetilde{s}_{0}:=R_{1}$ and according to the notation in (1.7) we can state the following result.

Proposition 6.4 Consider $p, q \in C^{1}([0,1], \mathbb{R})$ satisfying (1.2), one of the alternatives in (1.3)-(1.6), and (1.7)-(1.10). Fix $j \in\{0,1, \ldots, m-1\}, \mathcal{T} \in\left(\widetilde{s}_{j}, \widetilde{s}_{j+1}\right), \nu>0$ with $\widetilde{s}_{j}+\nu<\mathcal{T}$. Then,

$$
\begin{gathered}
\forall L_{1}>0 \quad \exists L_{2}>0: u_{d}(r)^{2}+u_{d}^{\prime}(r)^{2} \geq L_{1}, \quad \forall r \in\left[0, \widetilde{s}_{j}+\nu\right], \quad|d| \geq L_{2} \\
\Downarrow \\
\forall L>0 \quad \exists d_{L}>0: u_{d}(r)^{2}+u_{d}^{\prime}(r)^{2} \geq L, \quad \forall r \in\left[\widetilde{s}_{j}+\nu, \mathcal{T}\right], \quad|d| \geq d_{L} .
\end{gathered}
$$

Proof. Let us concentrate on the case $d>0$. The negative case can be treated in an analogous way. Arguing by contradiction, assume that there exists $L>0$ such that for every $n \in \mathbb{N}$ there exists $d_{n} \geq n$ and $t_{n} \in\left[\widetilde{s}_{j}+\nu, \mathcal{T}\right]$ such that

$$
u_{d_{n}}\left(t_{n}\right)^{2}+u_{d_{n}}^{\prime}\left(t_{n}\right)^{2}<L .
$$

Setting $\mathbf{v}_{n}:=\left(u_{d_{n}}\left(t_{n}\right), u_{d_{n}}^{\prime}\left(t_{n}\right)\right)$, we note that, up to a subsequence, $t_{n} \rightarrow \tau \in\left[\widetilde{s}_{j}+\nu, \mathcal{T}\right]$ and $\mathbf{v}_{n} \rightarrow \mathbf{d}$ with $|\mathbf{d}| \leq \sqrt{L}$. Consider now the solution $u_{0}$ of the Cauchy problem (4.20). Lemma 4.4 and Remark 4.7 guarantee that $u_{0}$ is defined in $\left[\widetilde{s}_{j}+\nu, \mathcal{T}\right]$, and it is unique too. Thus, by classical continuous dependence results (cf., for instance, [20]) we infer that $u_{d_{n}} \rightarrow u_{0}$ in $C^{1}\left(\left[\widetilde{s}_{j}+\nu, \mathcal{T}\right], \mathbb{R}\right)$ as $n \rightarrow+\infty$. Fixed $\epsilon>0$, from (6.14) we conclude that

$\exists L_{2}>0: u_{d_{n}}\left(\widetilde{s}_{j}+\nu\right)^{2}+u_{d_{n}}^{\prime}\left(\widetilde{s}_{j}+\nu\right)^{2} \geq u_{0}\left(\widetilde{s}_{j}+\nu\right)^{2}+u_{0}^{\prime}\left(\widetilde{s}_{j}+\nu\right)^{2}+\epsilon \quad \forall n \in \mathbb{N}, n \geq L_{2}$, a contradiction with the convergence of $u_{d_{n}}$ to $u_{0}$. This completes the proof.

The next step consists in extending the Elastic Property from a certain $\mathcal{T}_{0} \in\left(\widetilde{s}_{j}, \widetilde{s}_{j+1}\right)$ to $\widetilde{s}_{j+1}$, and even a bit further than $\widetilde{s}_{j+1}$. To this aim, we need to introduce some notation. According to (2.12), we set

$$
K:=\left(\frac{3}{2}+\frac{Q}{\delta+1}\right)^{\frac{1}{\gamma+1}}
$$

Moreover, define

$$
\sigma \in\left(0, \frac{R_{1}}{2}\right) \quad \text { and } \quad b=b(\sigma)>0: \quad b \leq \min \left\{\sigma^{\frac{1}{\gamma+1}}, \frac{1}{2}\right\} .
$$


Fix $j \in\{0,1, \ldots, m-1\}$. Since $p\left(\widetilde{s}_{j+1}\right)=p^{\prime}\left(\widetilde{s}_{j+1}\right)=0$ by (1.7), according also to (2.13) and (6.16), we deduce the existence of $\mathcal{T}_{0} \in\left(\widetilde{s}_{j}, \widetilde{s}_{j+1}\right)$ and $\nu_{0} \in\left(\widetilde{s}_{j+1}, \widetilde{s}_{j+2}\right)$ such that

$$
\begin{gathered}
r p^{\prime}(r)+N p(r)+N b>0 \quad \forall r \in\left[\mathcal{T}_{0}, \widetilde{s}_{j+1}+\nu_{0}\right], \\
\frac{p(r)+b}{\gamma+1} \leq \frac{1}{2} \quad \forall r \in\left[\mathcal{T}_{0}, \widetilde{s}_{j+1}+\nu_{0}\right], \\
\frac{R_{1}}{2} e^{-\max \left\{\frac{N-2}{R_{1}}+1+2 K, 1+\frac{2 Q^{\prime}}{\delta+1}\right\}\left(\widetilde{s}_{j+1}+\nu_{0}-\mathcal{T}_{0}\right)}>\sigma .
\end{gathered}
$$

For the above defined $\mathcal{T}_{0}$ and $\nu_{0}$, the following result holds.

Proposition 6.5 Under the assumptions of Proposition 6.4,

$$
\begin{gathered}
\forall L_{1}>0 \quad \exists L_{2}>0: u_{d}\left(\mathcal{T}_{0}\right)^{2}+u_{d}^{\prime}\left(\mathcal{T}_{0}\right)^{2} \geq L_{1}, \quad \forall d \in \mathbb{R} \text { with }|d| \geq L_{2} \\
\Downarrow \\
\forall L>0 \quad \exists d_{L}>0: u_{d}(r)^{2}+u_{d}^{\prime}(r)^{2} \geq L, \quad \forall r \in\left[\mathcal{T}_{0}, \widetilde{s}_{j+1}+\nu_{0}\right], \quad|d| \geq d_{L} .
\end{gathered}
$$

Proof. According to the previous notation, let us define

$$
\mathrm{E}_{u_{d}, b}(r):=r^{N}\left[\frac{1}{2}\left(u_{d}^{\prime}(r)^{2}+u_{d}(r)^{2}\right)+\frac{q(r)}{\delta+1}\left|u_{d}(r)\right|^{\delta+1}+\frac{p(r)+b}{\gamma+1}\left|u_{d}(r)\right|^{\gamma+1}\right] .
$$

We point out that

$$
\mathrm{E}_{u_{d}, b}(r) \leq \frac{R_{1}}{2} \quad \Longrightarrow \quad u_{d}(r) \leq 1, \quad u_{d}^{\prime}(r) \leq 1 \quad \forall r \in\left[\mathcal{T}_{0}, 1\right] \subset\left[R_{1}, 1\right] .
$$

From (4.5), it is immediate to verify that for every $r \in[0,1]$

$$
\begin{gathered}
\mathrm{E}_{u_{d}, b}^{\prime}(r)=r^{N-1}\left[-\frac{N-2}{2} u_{d}^{\prime}(r)^{2}+\frac{N}{2} u_{d}(r)^{2}+r u_{d}(r) u_{d}^{\prime}(r)\left(1+b\left|u_{d}(r)\right|^{\gamma-1}\right)+\right. \\
\left.+\frac{\left|u_{d}(r)\right|^{\delta+1}}{\delta+1}\left(r q^{\prime}(r)+N q(r)\right)+\frac{\left|u_{d}(r)\right|^{\gamma+1}}{\gamma+1}\left(r p^{\prime}(r)+N p(r)+N b\right)\right] .
\end{gathered}
$$

Assumption (6.20) guarantees the existence of $d_{R_{1}}>0$ such that $\mathrm{E}_{u_{d}, b}\left(\mathcal{T}_{0}\right)>R_{1}$ for every $d \in \mathbb{R}$ with $|d| \geq d_{R_{1}}$.

We claim that

$$
\mathrm{E}_{u_{d}, b}(r)>\sigma, \quad \forall r \in\left[\mathcal{T}_{0}, \widetilde{s}_{j+1}+\nu_{0}\right], \quad|d| \geq d_{R_{1}} .
$$

Suppose, by contradiction, that there exist $t_{0}, l_{0} \in\left(\mathcal{T}_{0}, \widetilde{s}_{j+1}+\nu_{0}\right]$ with $t_{0}<l_{0}$ and $d_{0} \in \mathbb{R}$ with $\left|d_{0}\right| \geq d_{R_{1}}$ such that

$$
\begin{gathered}
\sigma<\mathrm{E}_{u_{d_{0}}, b}(r)<\frac{R_{1}}{2}, \quad \forall r \in\left(t_{0}, l_{0}\right), \\
\mathrm{E}_{u_{d_{0}}, b}\left(t_{0}\right)=\frac{R_{1}}{2} \quad \text { and } \quad \mathrm{E}_{u_{d_{0}}, b}\left(l_{0}\right)=\sigma .
\end{gathered}
$$


From (6.22), we deduce that $u_{d_{0}}(r) \leq 1$ and $u_{d_{0}}^{\prime}(r) \leq 1$ for every $r \in\left[t_{0}, l_{0}\right]$. As a consequence, according to (2.13) and (6.17), we get

$$
\mathrm{E}_{u_{d_{0}}, b}^{\prime}(r) \geq-r^{N}\left[\frac{N-2+R_{1}}{2 R_{1}} u_{d_{0}}^{\prime}(r)^{2}+\frac{1}{2} u_{d_{0}}(r)^{2}+b\left|u_{d_{0}}^{\prime}(r)\right|\left|u_{d_{0}}(r)\right|^{\gamma}+\frac{u_{d_{0}}(r)^{2}}{\delta+1} Q^{\prime}\right] .
$$

Note that

$$
\left|u_{d_{0}}^{\prime}(r)\right| \leq\left|u_{d_{0}}(r)\right| \Longrightarrow\left|u_{d_{0}}^{\prime}(r)\right|\left|u_{d_{0}}(r)\right|^{\gamma} \leq\left|u_{d_{0}}(r)\right|^{\gamma+1} .
$$

Consider now the case $\left|u_{d_{0}}(r)\right| \leq\left|u_{d_{0}}^{\prime}(r)\right|$. Taking into account (6.18) and the energy definition, we observe that

$$
\sigma \leq \mathrm{E}_{u_{d_{0}}, b}(r) \leq \frac{1}{2} u_{d_{0}}^{\prime}(r)^{2}+\left(1+\frac{Q}{\delta+1}\right)\left|u_{d_{0}}(r)\right|^{\gamma+1}, \quad \forall r \in\left[t_{0}, l_{0}\right],
$$

from which it follows

$$
\sigma \leq \frac{1}{2} u_{d_{0}}^{\prime}(r)^{2}+\left(1+\frac{Q}{\delta+1}\right)\left|u_{d_{0}}^{\prime}(r)\right|^{\gamma+1} \leq\left(\frac{3}{2}+\frac{Q}{\delta+1}\right)\left|u_{d_{0}}^{\prime}(r)\right|^{\gamma+1} .
$$

Recalling the definition in (6.15), we have so proved that for every $r \in\left[t_{0}, l_{0}\right]$

$$
\left|u_{d_{0}}(r)\right| \leq\left|u_{d_{0}}^{\prime}(r)\right| \quad \Longrightarrow \quad\left|u_{d_{0}}^{\prime}(r)\right|\left|u_{d_{0}}(r)\right|^{\gamma} \leq\left|u_{d_{0}}^{\prime}(r)\right| \leq \frac{K\left|u_{d_{0}}^{\prime}(r)\right|^{2}}{\sigma^{\frac{1}{\gamma+1}}},
$$

which combined with (6.25) leads to

$\mathrm{E}_{u_{d_{0}}, b}^{\prime}(r) \geq-r^{N}\left[\left(\frac{N-2+R_{1}}{2 R_{1}}+\frac{b K}{\sigma^{\frac{1}{\gamma+1}}}\right) u_{d_{0}}^{\prime}(r)^{2}+\left(\frac{1}{2}+\frac{Q^{\prime}}{\delta+1}\right) u_{d_{0}}(r)^{2}+b\left|u_{d_{0}}(r)\right|^{\gamma+1}\right]$,

for every $r \in\left[t_{0}, l_{0}\right]$. Recalling the energy definition, we infer

$$
\mathrm{E}_{u_{d_{0}}, b}^{\prime}(r) \geq-\max \left\{\frac{N-2+R_{1}}{R_{1}}+\frac{2 b K}{\sigma^{\frac{1}{\gamma+1}}}, 1+\frac{2 Q^{\prime}}{\delta+1}, \gamma+1\right\} \mathrm{E}_{u_{d_{0}}, b}(r), \quad \forall r \in\left[t_{0}, l_{0}\right] .
$$

Taking into account (6.16) and our assumption $N \geq 3$, by Gronwall Lemma we deduce

$$
\mathrm{E}_{u_{d_{0}}, b}\left(l_{0}\right) \geq \mathrm{E}_{u_{d_{0}}, b}\left(t_{0}\right) e^{-\max \left\{\frac{N-2}{R_{1}}+1+2 K, 1+\frac{2 Q^{\prime}}{\delta+1}\right\}\left(l_{0}-t_{0}\right)},
$$

which, according to (6.24) and (6.19), implies

$$
\sigma \geq \frac{R_{1}}{2} e^{-\max \left\{\frac{N-2}{R_{1}}+1+2 K, 1+\frac{2 Q^{\prime}}{\delta+1}\right\}\left(l_{0}-t_{0}\right)} \geq \frac{R_{1}}{2} e^{-\max \left\{\frac{N-2}{R_{1}}+1+2 K, 1+\frac{2 Q^{\prime}}{\delta+1}\right\}\left(\widetilde{s}_{j+1}+\nu_{0}-\mathcal{T}_{0}\right)}>\sigma,
$$

a contradiction. Thus, the claim is proved and (6.23) is satisfied. In particular, there exists $\sigma_{1}>0$ such that

$$
u_{d}(r)^{2}+u_{d}^{\prime}(r)^{2}>\sigma_{1}, \quad \forall r \in\left[\mathcal{T}_{0}, \widetilde{s}_{j+1}+\nu_{0}\right], \quad|d| \geq d_{R_{1}} .
$$


The last step consists in proving that the Elastic property (6.21) follows by combining (6.20) with (6.26).

We argue exactly as in Proposition 6.4, focusing on the case $d>0$, and assuming, by contradiction, the existence of $L>0$ such that for every $n \in \mathbb{N}$ with $n \geq d_{R_{1}}$ there exists $d_{n} \geq n$ and $t_{n} \in\left[\mathcal{T}_{0}, \widetilde{s}_{j+1}+\nu_{0}\right]$ such that

$$
\sigma_{1}<u_{d_{n}}\left(t_{n}\right)^{2}+u_{d_{n}}^{\prime}\left(t_{n}\right)^{2}<L .
$$

Setting $\mathbf{v}_{n}:=\left(u_{d_{n}}\left(t_{n}\right), u_{d_{n}}^{\prime}\left(t_{n}\right)\right)$, notice that, up to a subsequence, $t_{n} \rightarrow \tau \in\left[\mathcal{T}_{0}, \widetilde{s}_{j+1}+\nu_{0}\right]$ and $\mathbf{v}_{n} \rightarrow \mathbf{d}$ with $|\mathbf{d}| \leq \sqrt{L}$. Taking into account Remark 4.7, let $u_{0}$ be the unique solution of the Cauchy problem (4.20), defined in $\left[\mathcal{T}_{0}, \widetilde{s}_{j+1}+\nu_{0}\right]$. Thus, by the classical continuous dependence results, $u_{d_{n}} \rightarrow u_{0}$ in $C^{1}\left(\left[\mathcal{T}_{0}, \widetilde{s}_{j+1}+\nu_{0}\right], \mathbb{R}\right)$ as $n \rightarrow+\infty$. Fixed $\epsilon>0$, from (6.20) we conclude that

$$
\exists L_{2}>0: \quad u_{d_{n}}\left(\mathcal{T}_{0}\right)^{2}+u_{d_{n}}^{\prime}\left(\mathcal{T}_{0}\right)^{2} \geq u_{0}\left(\mathcal{T}_{0}\right)^{2}+u_{0}^{\prime}\left(\mathcal{T}_{0}\right)^{2}+\epsilon, \quad \forall n \in \mathbb{N}, \quad n \geq L_{2},
$$

a contradiction. The Elastic property (6.21) is achieved.

By applying a finite number of times Proposition 6.4 and Proposition 6.5, combined with Proposition 6.1 we can conclude that

Proposition 6.6 Under the assumptions of Theorem 1.1, for every $L_{1}>0$ there exists $L_{2}>0$ such that, for every $d \in \mathbb{R}$ with $|d| \geq L_{2}, u_{d}$ is the unique solution of (2.2) and

$$
u_{d}(r)^{2}+u_{d}^{\prime}(r)^{2} \geq L_{1}, \quad \forall r \in[0,1] .
$$

\section{Rotation number and multiplicity of solutions}

This section is devoted to define the rotation number associated with the solution of the Cauchy problem (2.2), and to show how some estimates on the rotation number lead to multiplicity results.

Observe first that (2.2) can be equivalently written in the form

$$
\left\{\begin{array}{l}
r^{N-1} u^{\prime}(r)=y(r) \\
y^{\prime}(r)=-r^{N-1}|u(r)|^{\delta-1} u(r) q(r)-r^{N-1}|u(r)|^{\gamma-1} u(r) p(r) \\
(u(0), y(0))=(d, 0) .
\end{array}\right.
$$

For any $\mu>0$, introduce the polar coordinates $(\vartheta, \rho)$ by setting $\mu u=\rho \sin \vartheta, y=\rho \cos \vartheta$. Hence, for each interval $[s, t] \subset[0,1]$ and for each solution $u_{d}$ of $(2.2)$ defined on $[s, t]$ and such that

$$
u_{d}(r)^{2}+u_{d}^{\prime}(r)^{2}>0 \quad \forall r \in[s, t],
$$

we can define the rotation number

$$
\operatorname{Rot}_{[s, t]}\left(u_{d}\right):=\frac{\vartheta(t)-\vartheta(s)}{\pi},
$$


which counts the number of half-turns of the vector $\overrightarrow{\left((0,0),\left(u, u^{\prime}\right)\right)}$ as $r$ moves from $s$ to $t$. According to [14], the rotation number can be expressed by the following relation

$$
\operatorname{Rot}_{[s, t]}\left(u_{d}\right)=\frac{\mu}{\pi} \int_{s}^{t} \frac{r^{N-1} u_{d}^{\prime}(r)^{2}+r^{N-1}\left|u_{d}(r)\right|^{\delta+1} q(r)+r^{N-1}\left|u_{d}(r)\right|^{\gamma+1} p(r)}{r^{2(N-1)} u_{d}^{\prime}(r)^{2}+\mu^{2} u_{d}(r)^{2}} d r .
$$

Note that for every $j \in \mathbb{N}$

$$
\operatorname{Rot}_{[0,1]}\left(u_{d}\right)=\frac{1}{2}+j \quad \Longleftrightarrow \quad u_{d}(1)=0 \text { and } u_{d} \text { has exactly } j \text { zeros in }(0,1) .
$$

This relation is independent from the choice of $\mu>0$. For this reason we are allowed to estimate the rotation numbers with values in $\frac{1}{2}+\mathbb{N}$ by choosing $\mu>0$ according to our convenience.

Moreover, from (1.2) observe that if $\operatorname{Rot}_{[s, t]}\left(u_{d}\right)$ is well defined, then

$$
\left[s_{*}, t_{*}\right] \subset[s, t] \subset[0,1] \Longrightarrow \operatorname{Rot}_{\left[s_{*}, t_{*}\right]}\left(u_{d}\right) \leq \operatorname{Rot}_{[s, t]}\left(u_{d}\right) .
$$

Taking into account Remark 4.6 and Proposition 6.6, we know that in general the validity of condition (7.2) is not guaranteed for $t \geq \widetilde{s_{1}}$ and small values of $d$. For this reason, fixed a positive constant $c$, we introduce, inspired by [14], the map $\operatorname{Rot}_{c}^{*}:\left\{u_{d} \in C^{1}([0,1], \mathbb{R})\right.$ : $u_{d}$ solves $(2.2)$ with $\left.d \neq 0\right\} \rightarrow \mathbb{R}$ by setting

$$
\operatorname{Rot}_{c}^{*}\left(u_{d}\right):=\left\{\begin{array}{cl}
\operatorname{Rot}_{[0,1]}\left(u_{d}\right) & \text { if }(7.2) \text { holds in }[0,1] \text { and } \operatorname{Rot}_{[0,1]}\left(u_{d}\right) \leq c \\
c & \text { otherwise. }
\end{array}\right.
$$

The continuity of this function follows by combining the continuity of the Volterra integral operator associated to (7.1), the uniqueness result in Remark 4.6 with the following proposition

Proposition 7.1 Under the assumptions of Lemma 4.3, fix $d \neq 0$. Assume the existence of $\widetilde{s}_{j} \in(0,1]$ such that

$$
u_{d}(r)^{2}+u_{d}^{\prime}(r)^{2}>0, \quad \forall r \in\left[0, \widetilde{s}_{j}\right) \quad \text { and } \quad u_{d}\left(\widetilde{s}_{j}\right)^{2}+u_{d}^{\prime}\left(\widetilde{s}_{j}\right)^{2}=0 .
$$

Then,

$$
\operatorname{Rot}_{[0, t]}\left(u_{d}\right) \rightarrow+\infty \quad \text { as } t \rightarrow \widetilde{s}_{j}^{-} .
$$

Proof. As observed in (7.5), the map $t \mapsto \operatorname{Rot}_{[0, t]}\left(u_{d}\right)$ is nondecreasing. Hence, $\operatorname{Rot}_{[0, t]}\left(u_{d}\right)$ admits a limit $l \in(0, \overline{\mathbb{R}}]$ as $t \rightarrow \widetilde{s}_{j}^{-}$. Assume, by contradiction, that $l \in \mathbb{R}$. Then, $u_{d}$ and $u_{d}^{\prime}$ have a finite numer of zeros in $\left[0, \widetilde{s}_{j}\right)$. In particular, from (7.6), we deduce the existence of $r_{0} \in\left(0, \widetilde{s}_{j}\right)$ such that

$$
u_{d}(r) u_{d}^{\prime}(r)<0, \quad \forall r \in\left[r_{0}, \widetilde{s}_{j}\right) .
$$

Thus, according to (4.5) we infer that for every $r \in\left[r_{0}, \widetilde{s}_{j}\right)$

$$
\left(u_{d}^{\prime}(r)^{2}\right)^{\prime}=2 u_{d}^{\prime}(r) u_{d}^{\prime \prime}(r)=2\left|u_{d}(r)\right|^{\delta}\left|u_{d}^{\prime}(r)\right| q(r)+2\left|u_{d}(r)\right|^{\gamma}\left|u_{d}^{\prime}(r)\right| p(r)-\frac{2(N-1)}{r} u_{d}^{\prime}(r)^{2},
$$


which leads to

$$
\left(u_{d}^{\prime}(r)^{2}\right)^{\prime} \geq-\frac{2(N-1)}{r_{0}} u_{d}^{\prime}(r)^{2}, \quad \forall r \in\left[r_{0}, \widetilde{s}_{j}\right) .
$$

Finally, Gronwall Lemma and (7.7) enable us to conclude that

$$
u_{d}^{\prime}\left(\widetilde{s}_{j}\right)^{2} \geq e^{-\frac{2(N-1)}{r_{0}}\left(\widetilde{s}_{j}-\widetilde{t}\right)} u_{d}^{\prime}\left(r_{0}\right)^{2}>0,
$$

contradicting (7.6). This completes the proof.

We now present two theorems which allow us to provide the existence of solutions of the two-point boundary value problem (2.1) when uniqueness of the solutions of the associated Cauchy problem is not guaranteed. The first Theorem has been proved in [12], by applying a variant of the classical "Théorème Fondamental" of Leray and Schauder [24].

Theorem 7.2 [12] Let $F:[a, b] \times \mathbb{R}^{2} \rightarrow \mathbb{R}^{2}$ satisfy the Carathéodory conditions. Let $J \subset \mathbb{R}$ be a (non-degenerate) interval, let $\tau: J \rightarrow[a, b]$ and $\eta: J \rightarrow \mathbb{R}^{2}$ be two continuous functions such that, for each $d \in J$, all the (non-continuable) solutions to the Cauchy problem

$$
\left\{\begin{array}{l}
\dot{x}=F(r, x) \\
x(\tau(d))=\eta(d)
\end{array}\right.
$$

are defined on $[a, b]$. Then, there is a closed connected set $\mathcal{C} \subset J \times C\left([a, b], \mathbb{R}^{2}\right)$ such that the projection $\operatorname{Pr}_{1}$ of $\mathcal{C}$ to $J$ covers $J$ and, for every $(d, u) \in \mathcal{C}, u(\cdot)$ is a solution of $(7.8)$ defined on $[a, b]$.

Arguing on the same lines of [12], this theorem allows us to obtain, by means of estimates on rotation numbers, solutions to $(2.1)$ characterized by their nodal properties.

Theorem 7.3 Under the assumptions of Lemma 4.3, suppose that there exist $j \in \mathbb{N}$, $c \in \mathbb{R}$ with $c>j+1$ and two positive numbers $\mathcal{S}<\mathcal{R}$ such that

$$
\operatorname{Rot}_{c}^{*}\left(u_{d}\right)>\frac{1}{2}+j \quad\left(\text { resp. }<\frac{1}{2}+j\right), \quad \text { for each solution } u_{d} \text { of }(2.2) \text { with }|d|=\mathcal{S},
$$

as well as

$$
\operatorname{Rot}_{c}^{*}\left(u_{d}\right)<\frac{1}{2}+j \quad\left(\text { resp. }>\frac{1}{2}+j\right), \quad \text { for each solution } u_{d} \text { of }(2.2) \text { with }|d|=\mathcal{R} .
$$

Then, there are at least two solutions $u_{j}$ and $v_{j}$ of $(2.1)$ with $u_{j}(0) \in(\mathcal{S}, \mathcal{R})$ and $v_{j}(0) \in$ $(-\mathcal{R},-\mathcal{S})$ having exactly $j$ zeros in $(0,1)$.

Proof. Let us study the following problem

$$
\left\{\begin{array}{l}
u^{\prime}(r)=r^{-(N-1)} y(r) \\
y^{\prime}(r)=-r^{N-1}|u(r)|^{\delta-1} u(r) q(r)-r^{N-1}|u(r)|^{\gamma-1} u(r) p(r) \\
(u, y)\left(r_{d}\right)=\eta(d),
\end{array}\right.
$$


where $r_{d}$ is introduced in Lemma 3.2 and $\eta:[\mathcal{S}, \mathcal{R}] \longrightarrow \mathbb{R}^{2} \backslash\{0\}$ is the function defined by $\eta(d)=\left(u_{d}\left(r_{d}\right), r_{d}^{N-1} u_{d}^{\prime}\left(r_{d}\right)\right)$, with $u_{d}$ solution to (2.2). Due to the uniqueness of $u_{d}$ in $\left[0, r_{d}\right]$ stated by Lemma 3.3 combined with the classical results on continuous dependence from initial data (cf., for instance, [20]), we easily deduce the continuity of the map $\tau: d \mapsto r_{d}$ and of the map $\eta$. Note that $\tau([\mathcal{S}, \mathcal{R}])$ is a closed interval contained in $\left(0, R_{1}\right)$. Denote by $a$ its left extreme. Lemma 4.3 ensures that all the solutions to (7.9) are defined in $[a, 1]$. Thus, we can apply Theorem 7.2 to deduce the existence of a closed connected set $\mathcal{C} \subset[\mathcal{S}, \mathcal{R}] \times C\left([a, 1], \mathbb{R}^{2}\right)$ such that $\operatorname{Pr}_{1}(\mathcal{C})=[\mathcal{S}, \mathcal{R}]$ and, for every $(d, z) \in \mathcal{C}, z=(u, w)$ is a solution of $(7.9)$ defined on $[a, 1]$. Lemma 3.3 guarantees that this solution $z=(u, w)$ can be extended to the whole interval $[0,1]$, and its first component $u=u_{d}$ solves the Cauchy problem (2.2).

As a consequence, there exist two solutions $u_{\mathcal{S}}$ and $u_{\mathcal{R}}$ whose rotation numbers satisfy, by assumption,

$$
\operatorname{Rot}_{c}^{*}\left(u_{\mathcal{S}}\right)>\frac{1}{2}+j \quad\left(\operatorname{resp} .<\frac{1}{2}+j\right), \quad \operatorname{Rot}_{c}^{*}\left(u_{\mathcal{R}}\right)<\frac{1}{2}+j \quad\left(\text { resp. }>\frac{1}{2}+j\right) .
$$

Note that $\operatorname{Pr}_{2}(\mathcal{C})$ is a connected set in $C\left([a, 1], \mathbb{R}^{2}\right)=C([a, 1], \mathbb{R}) \times C([a, 1], \mathbb{R})$. Hence, $\operatorname{Pr}_{2}^{\prime}(\mathcal{C}):=\left\{u_{d} \in C^{1}([a, 1], \mathbb{R}): u_{d}\right.$ solves $\left.(2.2)\right\}$ is connected too. From Lemma 3.3, we easily deduce that

$$
\begin{aligned}
\widetilde{\mathcal{C}_{2}} & :=\left\{u_{d} \in C^{1}([0,1], \mathbb{R}): u_{\left.d\right|_{[a, 1]}} \in \operatorname{Pr}_{2}^{\prime}(\mathcal{C})\right\} \subset \\
& \subset\left\{u_{d} \in C^{1}([0,1], \mathbb{R}): u_{d} \text { solves }(2.2), d \in[\mathcal{S}, \mathcal{R}]\right\}
\end{aligned}
$$

is still a connected set. Taking into account the continuity of the operator $\operatorname{Rot}_{c}^{*}(\cdot)$ in $\widetilde{\mathcal{C}_{2}}$, the fact that $u_{\mathcal{S}}, u_{\mathcal{R}} \in \widetilde{\mathcal{C}}_{2}$ and satisfy $(7.10)$, by the intermediate value theorem we infer the existence of a solution $u_{d} \in \widetilde{\mathcal{C}}_{2}$ with $d \in(\mathcal{S}, \mathcal{R})$ and such that $\operatorname{Rot}_{c}^{*}\left(u_{d}\right)=\frac{1}{2}+j$. Since $\frac{1}{2}+j<c$, we get $\operatorname{Rot}_{[0,1]}\left(u_{d}\right)=\frac{1}{2}+j$. Thus, (7.4) ensures that $u_{d}$ solves the twopoint boundary value problem $(2.1)$ and it has exactly $j$ zeros in $(0,1)$. Furthermore, $u_{d}(0)=d \in(\mathcal{S}, \mathcal{R})$.

To complete the proof, it remains to prove the existence of at least a solution $v_{j}$ to $(2.1)$ with $v_{j}(0)<0$, having exactly $j$ zeros in $(0,1)$. To this aim, it is sufficient to redefine the map $\eta$ in $[-\mathcal{R},-\mathcal{S}]$, and, arguing exactly as in the previous step, we easily achieve the thesis.

\section{Estimates on the rotation numbers}

Proposition 6.6 ensures the existence of $L_{1}>0, \mathcal{L}>0$ and $j_{0} \in \mathbb{N}$ such that $\left\|u_{d}\right\|_{1}^{2} \geq L_{1}$ for every $|d| \geq \mathcal{L}, \operatorname{Rot}_{[0,1]}\left(u_{ \pm \mathcal{L}}\right)$ is well defined, and

$$
\operatorname{Rot}_{[0,1]}\left(u_{ \pm \mathcal{L}}\right)<\frac{1}{2}+j_{0}
$$

Observe that formula (8.1) holds for every choice of $\mu>0$ in the definition of rotation number. 
The following propositions provide lower bounds on the rotation numbers of the solutions to (2.2) when $|d|$ is both sufficiently large and sufficiently small.

Proposition 8.1 Under the assumptions of Theorem 1.1, let consider $j \in \mathbb{N}$ with $j \geq j_{0}$. Then, there exists $\mathcal{R}>\mathcal{L}$ such that for every $d \in \mathbb{R}$ with $|d| \geq \mathcal{R}$ and for every solution $u_{d}$ of $(2.2), \operatorname{Rot}_{[0,1]}\left(u_{d}\right)$ is well defined and

$$
\operatorname{Rot}_{[0,1]}\left(u_{d}\right)>\frac{1}{2}+j .
$$

Proof. The rotation number is well defined due to Proposition 6.6.

Consider now $R_{0} \in\left(0, R_{1}\right)$ as in Remark 2.1, and fix

$$
\mu=\mu(j)>\left(\frac{1}{2}+j\right) \frac{\pi R_{1}^{2(N-1)}}{R_{0}^{N-1}\left(R_{1}-R_{0}\right)} .
$$

According to (7.5), (1.2) and (2.9), we obtain

$$
\operatorname{Rot}_{[0,1]}\left(u_{d}\right) \geq \operatorname{Rot}_{\left[R_{0}, R_{1}\right]}\left(u_{d}\right) \geq \frac{\mu R_{0}^{N-1}}{\pi} \int_{R_{0}}^{R_{1}} \frac{u_{d}^{\prime}(r)^{2}+\left|u_{d}(r)\right|^{\delta+1} q_{0}}{R_{1}^{2(N-1)} u_{d}^{\prime}(r)^{2}+\mu^{2} u_{d}(r)^{2}} d r .
$$

Since $\delta>1$, there exists $H_{\mu}>0$ such that

$$
R_{1}^{2(N-1)}\left|u_{d}(r)\right|^{\delta+1} q_{0} \geq \mu^{2} u_{d}(r)^{2}-H_{\mu} .
$$

In particular, taking into accout (8.2), we can find $\epsilon>0$ such that

$$
\begin{aligned}
\operatorname{Rot}_{[0,1]}\left(u_{d}\right) & \geq \frac{\mu R_{0}^{N-1}\left(R_{1}-R_{0}\right)}{\pi R_{1}^{2(N-1)}}-\frac{\mu R_{0}^{N-1}}{\pi R_{1}^{2(N-1)}} \int_{R_{0}}^{R_{1}} \frac{H_{\mu}}{R_{1}^{2(N-1)} u_{d}^{\prime}(r)^{2}+\mu^{2} u_{d}(r)^{2}} d r \geq \\
& >\frac{1}{2}+j+\epsilon-\frac{\mu R_{0}^{N-1}}{\pi R_{1}^{2(N-1)}} \int_{R_{0}}^{R_{1}} \frac{H_{\mu}}{R_{1}^{2(N-1)} u_{d}^{\prime}(r)^{2}+\mu^{2} u_{d}(r)^{2}} d r .
\end{aligned}
$$

We now apply Proposition 6.1 to conclude that there exists $\mathcal{R}=\mathcal{R}(\mu, j)>\mathcal{L}$ such that for every $d \in \mathbb{R}$ with $|d| \geq \mathcal{R}$

$$
\frac{\mu R_{0}^{N-1}}{\pi R_{1}^{2(N-1)}} \int_{R_{0}}^{R_{1}} \frac{H_{\mu}}{R_{1}^{2(N-1)} u_{d}^{\prime}(r)^{2}+\mu^{2} u_{d}(r)^{2}} d r \leq \epsilon,
$$

from which the thesis follows.

Proposition 8.2 Under the assumptions of Theorem 1.2, let consider $j \in \mathbb{N}$ with $j \geq j_{0}$ and $c \in \mathbb{R}$ with $c>j+1$. Then, there exists $\mathcal{S} \in(0, \mathcal{L})$ such that for every $d \in \mathbb{R} \backslash\{0\}$ with $|d| \leq \mathcal{S}$ every solution $u_{d}$ of $(2.2)$ is such that

$$
\operatorname{Rot}_{c}^{*}\left(u_{d}\right)>\frac{1}{2}+j .
$$


Proof. Due to the definition of $\operatorname{Rot}_{c}^{*}$, note that, for every $d \in \mathbb{R} \backslash\{0\}$, (8.3) is satisfied whenever the rotation number of $u_{d}$ is not defined in $[0,1]$.

Thus, let us focus on the solutions $u_{d}$ which verify

$$
u_{d}(r)^{2}+u_{d}^{\prime}(r)^{2}>0 \quad \forall r \in[0,1] .
$$

To complete the proof, it remains to show that there exists $\mathcal{S} \in(0, \mathcal{L})$ such that for every $d \in \mathbb{R} \backslash\{0\}$ with $|d| \leq \mathcal{S}$ and for every solution $u_{d}$ satisfying (8.4),

$$
\operatorname{Rot}_{[0,1]}\left(u_{d}\right)>\frac{1}{2}+j .
$$

To this aim, consider again $R_{0} \in\left(0, R_{1}\right)$ as in Remark 2.1 and $\mu$ satisfying (8.2). According to (7.5), (1.2) and (2.9), we obtain

$$
\operatorname{Rot}_{[0,1]}\left(u_{d}\right) \geq \operatorname{Rot}_{\left[R_{0}, R_{1}\right]}\left(u_{d}\right) \geq \frac{\mu R_{0}^{N-1}}{\pi} \int_{R_{0}}^{R_{1}} \frac{u_{d}^{\prime}(r)^{2}+\left|u_{d}(r)\right|^{\gamma+1} p_{0}}{R_{1}^{2(N-1)} u_{d}^{\prime}(r)^{2}+\mu^{2} u_{d}(r)^{2}} d r .
$$

Recalling that $\gamma<1$, we can apply Lemma 5.1 which guarantees the existence of $\mathcal{S}=$ $\mathcal{S}(\mu, j) \in(0, \mathcal{L})$ such that

$$
R_{1}^{2(N-1)}\left|u_{d}(r)\right|^{\gamma+1} p_{0} \geq \mu^{2} u_{d}(r)^{2} \quad \forall r \in\left[0, R_{1}\right], \quad \forall d \in \mathbb{R} \backslash\{0\}:|d| \leq \mathcal{S} .
$$

In particular, according to (8.2) we infer that for each $d \in \mathbb{R} \backslash\{0\}$ with $|d| \leq \mathcal{S}$

$$
\operatorname{Rot}_{[0,1]}\left(u_{d}\right) \geq \frac{\mu R_{0}^{N-1}\left(R_{1}-R_{0}\right)}{\pi R_{1}^{2(N-1)}}>\frac{1}{2}+j,
$$

from which the thesis follows.

\section{The main results}

This section is devoted to prove our main results. First, we concentrate on the proof of Theorem 1.1. To this aim, we wish to demonstrate the following result

Theorem 9.1 Under the assumption of Theorem 1.1, there exists $j_{0} \in \mathbb{N}$ such that for every integer $j \geq j_{0}$ there exist two radial solutions $u_{j}$ and $v_{j}$ of (2.1) having exactly $j$ zeros in $(0,1)$, satisfying $u_{j}(0)>0>v_{j}(0)$ and

$$
\lim _{j \rightarrow+\infty}\left\|u_{j}\right\|_{1}=+\infty, \quad \lim _{j \rightarrow+\infty}\left\|v_{j}\right\|_{1}=+\infty .
$$

Proof. Consider $L_{1}, \mathcal{L}$ and $j_{0} \in \mathbb{N}$ as in (8.1). Take $j \in \mathbb{N}$ with $j \geq j_{0}$ and $c \in \mathbb{R}$ with $c>j+1$. By (8.1), we observe that

$$
\operatorname{Rot}_{c}^{*}\left(u_{d}\right)=\operatorname{Rot}_{[0,1]}\left(u_{d}\right)<\frac{1}{2}+j_{0} \leq \frac{1}{2}+j, \quad \text { as }|d|=\mathcal{L} .
$$


From Proposition 8.1 combined with the definition of Rot $_{c}^{*}$, we deduce the existence of $\mathcal{R}>\mathcal{L}$ such that

$$
\operatorname{Rot}_{c}^{*}\left(u_{d}\right)>\frac{1}{2}+j, \quad \text { as }|d|=\mathcal{R} .
$$

We apply Theorem 7.3 with $\mathcal{S}=\mathcal{L}$ to conclude the existence of at least two solutions $u_{j}$ and $v_{j}$ of $(2.1)$ with $u_{j}(0)>\mathcal{L}>0>-\mathcal{L}>v_{j}(0)$ having exactly $j$ zeros in $(0,1)$. In particular, $\left\|u_{j}\right\|_{1}^{2} \geq L_{1},\left\|v_{j}\right\|_{1}^{2} \geq L_{1}$ and $\operatorname{Rot}_{[0,1]}\left(u_{j}\right)=\operatorname{Rot}_{[0,1]}\left(v_{j}\right)=j+1 / 2$. Combining these relations with the definition of rotation number given in (7.3), we deduce (9.1).

We are finally interested in proving Theorem 1.2, whose statement can be rewritten as follows.

Theorem 9.2 Under the assumption of Theorem 1.2, there exists $j_{0} \in \mathbb{N}$ such that for every integer $j \geq j_{0}$ there exist two radial solutions $z_{j}$ and $w_{j}$ of (2.1) having exactly $j$ zeros in $(0,1)$, satisfying $z_{j}(0)>0>w_{j}(0)$.

Proof. Consider $L_{1}, \mathcal{L}$ and $j_{0} \in \mathbb{N}$ as in (8.1). Take $j \in \mathbb{N}$ with $j \geq j_{0}$ and $c \in \mathbb{R}$ with $c>j+1$. By (8.1), we deduce (9.2). Moreover, from Proposition 8.2, we get the existence of $\mathcal{S} \in(0, \mathcal{L})$ such that

$$
\operatorname{Rot}_{c}^{*}\left(u_{d}\right)>\frac{1}{2}+j, \quad \text { as }|d|=\mathcal{S} .
$$

We apply Theorem 7.3 with $\mathcal{R}=\mathcal{L}$ to conclude the existence of at least two solutions $z_{j}$ and $w_{j}$ of $(2.1)$ with $z_{j}(0)>\mathcal{S}>0>-\mathcal{S}>w_{j}(0)$ having exactly $j$ zeros in $(0,1)$. Notice that these solutions differ from the ones obtained by the previous theorem, since Theorem 7.3 establishes that $z_{j}(0)<\mathcal{L}$ and $w_{j}(0)>-\mathcal{L}$.

Remark 9.3 For the sake of simplicity we have assumed (1.3)-(1.6) to describe the local behaviour of the functions $q$ and $p$ in a right neighbourhood of 0 . We point out that different assumptions on the behaviour of $q$ and $p$ next to 0 can be equivalently considered to obtain our main results. For instance, the weights $q$ and $p$ can be also required to respectively satisfy one of the following alternatives

$$
\begin{array}{rlrl}
\exists h>1, \beta>0: & q(r) \sim-\beta r^{h} \log r \quad \text { or } & q(r) \sim \beta e^{-\frac{1}{r}} & \text { as } r \rightarrow 0^{+}, \\
\text {and } & & \\
\exists k>1, \alpha>0: & p(r) \sim-\alpha r^{k} \log r \quad \text { or } \quad p(r) \sim \alpha e^{-\frac{1}{r}} & \text { as } r \rightarrow 0^{+} .
\end{array}
$$

Condition (1.4) can be replaced with the first alternative in (9.3) in the statements of our main theorems.

On the other hand, if the second alternative in (9.3) holds (i.e. if $q(r) \sim \beta e^{-\frac{1}{r}}$ as $\left.r \rightarrow 0^{+}\right)$no upper bounds on $\delta$ should be imposed to get our multiplicity results (neither assumption (1.12) nor (1.13) are needed in the statements of our main results). 


\section{References}

[1] I. Addou, A. Benmezaï, S.M. Bouguima and M. Derhab, Exactness results for generalized Ambrosetti-Brezis-Cerami problem and related one-dimensional elliptic equations, Electron. J. Differential Equations 66 (2000), 1-34.

[2] S. Alama Semilinear elliptic equations with sublinear indefinite nonlinearities, Adv. Differential Equations 4 (1999), 813-842.

[3] A. Ambrosetti, H. Brezis and G. Cerami, Combined effects of concave and convex nonlinearities in some elliptic problems, J. Funct. Anal. 122 (1994), 519543.

[4] G. Anello, On the Dirichlet problem for the equation $-\Delta u=g(x, u)+\lambda f(x, u)$ with no growth conditions on $f$, Taiwanese J. Math. 10 (2006), 1515-1522.

[5] M. Badiale, Infinitely many solutions for some indefinite nonlinear elliptic problems, Comm. Appl. Nonlinear Anal. 3 (1996), 61-76.

[6] T. Bartsch and M. Willem, Infinitely many radial solutions of a semilinear elliptic problem on $\mathbb{R}^{N}$, Arch. Rational Mech. Anal. 124 (1993), 261-276.

[7] K.J. Brown and T.F. Wu, A fibering map approach to a semilinear elliptic boundary value problem, Electron. J. Differential Equations 69 (2007), 1-9.

[8] G.J. Butler, Rapid oscillation, nonextendability, and the existence of periodic solutions to second order nonlinear ordinary differential equations, J. Differential Equations 22 (1976), 467-477.

[9] A. Capietto, W. Dambrosio and F. Zanolin, Infinitely many radial solutions to a boundary value problem in a ball, Ann. Mat. Pura Appl. 179 (2001), 159-188.

[10] A. Castro and A. Kurepa, Infinitely many radially symmetric solutions to a superlinear Dirichlet problem in a ball, Proc. Amer. Math. Soc. 101 (1987), 57-64.

[11] Y. Cheng, On an open problem of Ambrosetti, Brezis and Cerami, Differential Integral Equations 15 (2002), 1025-1044.

[12] F. Dalbono and F. Zanolin, Multiplicity results for asymptotically linear equations, using the rotation number approach, Mediterr. J. Math. 4 (2007), 127-149.

[13] W. Dambrosio, On the multiplicity of radial solutions to superlinear Dirichlet problems in bounded domains, J. Differential Equations 196 (2004), 91-118.

[14] C. Fabry and P. Habets, Periodic solutions of second order differential equations with superlinear asymmetric nonlinearities, Arch. Math. 60 (1993), 266-276. 
[15] D.G. De Figueiredo, J.P. Gossez and P. Ubilla, Local superlinearity and sublinearity for indefinite semilinear elliptic problems J. Funct. Anal. 199 (2003), 452-467.

[16] D.G. de Figueiredo, J.P. Gossez and P. Ubilla, Multiplicity results for a family of semilinear elliptic problems under local superlinearity and sublinearity, $J$. Eur. Math. Soc. 8 (2006), 269-286.

[17] F.O. DE PAIva, Positive solutions of elliptic problems with sublinear indefinite nonlinearity, preprint 2010.

[18] M. Delgado and A. SuÀrez, Positive solutions for the degenerate logistic indefinite superlinear problem: the slow diffusion case, Houston J. Math. 29 (2003), 801-820.

[19] M. García Huidobro, R. Manásevich and F. Zanolin, Infinitely many solutions for a Dirichlet problem with a nonhomogeneous $p$-Laplacian-like operator in a ball, Adv. Differencial Equation 2 (1997), 203-230.

[20] P. Hartman, Ordinary Differential Equations, Second Edition, Birkhäuser, Boston, 1982.

[21] Y. IL'YASOv, On nonlocal existence results for elliptic equations with convexconcave nonlinearities, Nonlinear Anal. 61 (2005), 211-236.

[22] Z. Jin, Multiple solutions for a class of semilinear elliptic equations, Proc. Amer. Math. Soc. 125 (1997), 3659-3667.

[23] S.T. Kyritsi and N.S. Papageorgiou, Pairs of positive solutions for $p$-Laplacian equations with combined nonlinearities, Commun. Pure Appl. Anal. 8 (2009), 10311051.

[24] J. Leray and J. Schauder, Topologie et équations fonctionnelles, Ann. Sci. Ecol. Norm. Sup. (3) 51 (1934), 45-78.

[25] S.J. Li and Z. LiU, Perturbations from symmetric elliptic boundary value problems, J. Differential Equations 185 (2002), 271-280.

[26] S.J. LI and Z. LIU, Multiplicity of solutions for some elliptic equations involving critical and supercritical Sobolev exponents, Topol. Methods Nonlinear Anal. 28 (2006), 235-261.

[27] S. Li, S.P. Wu and H.S. Zhou, Solutions to semilinear elliptic problems with combined nonlinearities, J. Differential Equations 185 (2002) 200-224.

[28] Y. Li, Z. LiU and C. ZHAO, Nodal solutions of a perturbed elliptic problem, Topol. Methods Nonlinear Anal. 32 (2008), 49-68. 
[29] Z. Liv, Exact number of solutions of a class of two-point boundary value problems involving concave and convex nonlinearities, Nonlinear Anal. 46 (2001), 181-197.

[30] S. LiU and S.J. LI, An elliptic equation with concave and convex nonlinearities, Nonlinear Anal. 53 (2003), 723-731.

[31] Z.L. LiU and J.B. Su, Solutions of some nonlinear elliptic problems with perturbation terms of arbitrary growth, Discrete Contin. Dyn. Syst. 10 (2004), 617-634.

[32] V. Moroz, On the Morse critical groups for indefinite sublinear elliptic problems, Nonlinear Anal. 52 (2003), 1441-1453.

[33] M. Naito and Y. NAito, Solutions with prescribed numbers of zeros for nonlinear second order differential equations, Funkcial. Ekvac. 37 (1994), 505-520.

[34] D. O'REgan and H. WAng, On the number of positive solutions of elliptic systems, Math. Nachr. 280 (2007), 1417-1430.

[35] W. Reichel and W. Walter, Radial solutions of equations and inequalities involving the $p$-Laplacian, J. Inequal. Appl. 1 (1997), 47-71.

[36] N.S. Papageorgiou and E.M. Rocha, A multiplicity theorem for a variable exponent Dirichlet problem, Glasg. Math. J. 50 (2008), 335-349.

[37] N.S. Papageorgiou and E.M. Rocha, Pairs of positive solutions for $p$-Laplacian equations with sublinear and superlinear nonlinearities which do not satisfy the AR-condition, Nonlinear Anal. 70 (2009), 3854-3863.

[38] P.H. Rabinowitz, Multiple critical points of perturbed symmetric functionals, Trans. Amer. Math. Soc. 272 (1982), 753-769.

[39] M. Ramos and H. Tehrani, Perturbation from symmetry for indefinite semilinear elliptic equations, Manuscripta Math. 128 (2009), 297-314.

[40] Z.Q. WANG, Nonlinear boundary value problems with concave nonlinearities near the origin, NoDEA Nonlinear Differential Equations Appl. 8 (2001), 15-33.

[41] T.F. Wu, On semilinear elliptic equations involving concave-convex nonlinearities and sign-changing weight function, J. Math. Anal. Appl. 318 (2006), 253-270.

[42] W. Zou, Variant fountain theorems and their applications, Manuscripta Math. 104 (2001), 343-358. 